\title{
A CONSERVATIVE STABLE FINITE ELEMENT METHOD FOR STOKES FLOW AND NEARLY INCOMPRESSIBLE LINEAR ELASTICITY ON RECTANGULAR GRID
}

\author{
YUYAN CHEN AND SHUO ZHANG
}

\begin{abstract}
Авstract. In this paper, we discuss finite element methods for the incompressible Stokes problem and the nearly incompressible linear elasticity problem. Specifically, we present a finite element pair for the incompressible Stokes problem, which satisfies the discrete inf-sup condition and the discrete Korn's inequality, and moreover, which is element-wise conservative. The pair provides a lockingfree method for the nearly incompressible linear elasticity problem without reduced integration.
\end{abstract}

\section{INTRODUCTION}

In this paper, we study the finite element method for the incompressible Stokes flow and the nearly incompressible linear elasticity problem of displacement formulation. The two problems are important models constructed in fluid and solid mechanics, and finding robust numerical schemes for solving the models has been of great interest due to the wide application of them in applied sciences. As the linear elasticity model can be rewritten into its mixed formulation as a perturbed Stokes problem with a virtual pressure, in this paper, we will study the stable finite element pair of the Stokes flow, and then apply it to the mixed and primal formulation of the linear elasticity. Specifically, we consider finite element (pair) that satisfies the three stability conditions, which are, with the detailed technical description given in Section 2,

SC1: the inf-sup condition hold uniformly;

SC2: the coercive condition, namely the discrete Korn's inequality, holds uniformly;

SC3: the divergence-free constraint is imposed in a primal formulation.

The first and the second conditions fall into the classical theory of Stokes problem, as it provides a necessary and sufficient condition for the well-posedness of the discrete problem; see, e.g., $[7,12,21,40]$. Also, computationally, a nearly incompressible material is well known to be problematic upon a direct application of a standard low order finite element method. This locking phenomenon can be circumvented when a finite element pair that satisfies SC 1 and SC $\mathbf{2}$ used for the mixed formulation of the nearly-incompressible linear elasticity problem. The third condition is sometimes known as mass conservation and being more and more clearly recognized recently.

2000 Mathematics Subject Classification. 65M60, 76M10.

Key words and phrases. incompressible Stokes problem, nearly incompressible linear elasticity problem, stable finite element, conservative. 
Though it is not yet fully revealed how methods that enforce divergence-free would be superior to those that do not, it has been observed that satisfying the property can avoid possible instabilities that can arise from violation of mass conservation [5,31,56,57]. Moreover, in any pair that satisfies SC 3, the pressure space is the primal divergence of the velocity space, and it will be, in some sense, the biggest space that can make the inf-sup condition hold combined with the given velocity(displacement) space. Hence the capacity of the velocity(displacement) space can be fully exploited by such a pair. This way, the condition SC 3 is desirable in many applications. Besides, for pairs that satisfy SC 1 and SC 3, a discrete Stokes complex can usually be expected, the importance of which has been more and more clear; see [37,72] and the references therein for some examples. For linear elasticity problem, SC 3 can help avoid the reduced integration in the divergence part of the bilinear form.

There have been many kinds of finite element pairs reported which satisfy part of or all of the conditions. Compared to the conforming finite element pairs(see, e.g., [63]), to ensure condition SC 1, nonconforming finite element pairs (see, e.g., [27, 36, 68]) can admit bigger flexibility in designing and implementing. However, when nonconforming elements are used, a discrete version of the Korn's inequality may fail which plays a key role for the coercivity of the bilinear form. Though some nonconforming elements admit a discrete Korn's inequality, (c.f., e.g., [20, 69]), some others will not(c.f., e.g., [19,37,52]). This leads to an essential obstacle when the traction boundary condition is considered. As the rectangular grids are widely used where the problem geometry is of rectangular nature, in this paper, we study nonconforming finite element pairs on rectangular grids which satisfy SC 1, SC 2 and particularly SC 3. Indeed, among the finite elements reported to satisfy the conditions SC 1, SC 2 and SC 3, such as elements in [4, 32, 38, 42, $49,58,61,63,64,70,73]$, no nonconforming one is known to us on rectangular grids.

We remark that, there have been various approaches utilised in regard to the conditions, such as the discontinuous Galerkin methods [24,28-30], the isogeometric methods [33-35], the least square finite element methods $[11,14,26,43]$, finite element methods with enhanced stabilisation $[9,13,23,25,62]$, the element free Galerkin method [10, 67], stabilised finite element with postprocessing [8], and the NURBS method [6,50]. They provide various approaches to construct a divergence-free velocity field for Stokes problems. In this paper, we focus ourselves on the traditional finite element methods, and will not mention other approaches too much.

In this paper, we develop a finite element pair $\left(\boldsymbol{V}^{\mathrm{LTZ}, \mathrm{bl} \pm}-\mathbb{P}_{1}\right)$ that satisfies $\mathbf{S C} \mathbf{1}$, SC 2 and SC 3 in a nonconforming way. Specifically, we use the Lin-Tobiska-Zhou element [55] for one component of the velocity, and an enhanced bilinear element for the other; for pressure, we use the discontinuous piecewise linear polynomial space. The enhanced bilinear element functions are not continuous, but its first order moment, namely the integration of the function multiplicated with linear polynomials, is continuous across internal edges. This characteristic plays a crucial 
role in constructing SC 1 and SC 2. Moreover, the pair presented in this paper would be believed to be among ones of lowest degree that satisfy SC 1, SC 2 and SC 3 simultaneously on rectangular grids. Indeed, if we use piecewise constant functions for pressure, there is no finite element pair that satisfies generally SC 1, SC 2 and SC 3 known to us. Particularly, the continuous linear element space on rectangular grids is trivial, and the vector nonconforming $P_{1}$ element space on rectangular grids combined with piecewise constant space forms an unstable pair [47,51]. ${ }^{1}$

Our element pair is similar to the one presented by Kouhia-Stenberg ( [53], 1995). The KouhiaStenberg's element pair uses the conforming and nonconforming $P_{1}$ polynomial space for the two components of the velocity (displacement), respectively, and the piecewise constant space for the pressure on triangular grids. The pair satisfies SC 1, SC 2 and SC 3 simultaneously, and it is believed to be one of lowest degree. Its success illustrated that when different finite elements are used for each component, a balance between flexibility and continuity can be expected. The same methodology then finds its application on other pairs. Ming-Shi [59] constructed a pair which uses the bilinear element and the rotated bilinear element for velocity, and piecewise constant for pressure. Several other similar element pairs can be found constructed in González-Galván [41]. For these pairs mentioned, conditions SC 1 and SC $\mathbf{2}$ are verified, while condition SC 3 is not. The velocity space considered in the present paper can be viewed as the velocity space in MingShi [59] enriched with bubble-like functions for stabilisation. The stabilization mechanism here is different from that of, e.g., [15] or [2] where multilevel enrichment is hired.

The finite element pair in this paper is constructed on rectangular grids. The analysis can be repeated on a parallelogram grid that can be transferred to a rectangular grid by an affine mapping; however, it is not easy to construct a finite element pair on general quadrilateral grids. For one thing, for other parallelogram grids rather than ones mentioned above, it is difficult to carry out the Stenberg's macroelement argument for the stability conditions SC $\mathbf{1}$ and SC 2, let alone the general quadrilateral grids. For another, in general, the intrinsic symmetry of the grid which comes from the tensor product characteristic of the rectangle grid will lose for general quadrilateral grids. Therefore the continuity of the finite element space, which would be easy to obtain on rectangle grids due to the symmetry, can not generally be preserved on quadrilateral grids. This makes the discrete Korn's inequality an issue. The only finite element pair that satisfies both SC 1 and SC 3 known to us is the one defined in [72], but the discrete Korn's inequality is open.

The remaining of the paper is organised as follows. In Section 2, we give the description of the model problem and the finite element. Specifically, we present and proved partially the main result of the paper. In Section 3, we construct a conforming pair that satisfies SC 1 and SC 2, which is an auxiliary one for the mathematical analysis of the $V^{\mathrm{LTZ}, \mathrm{bl}}-\mathbb{P}_{1}$ pair. Then in Section

\footnotetext{
${ }^{1}$ We remark that $\mathrm{Hu}$ [45] and Hu and Shi [48] constructed discrete Korn's inequality on vector nonconforming $P_{1}$ element space on rectangle domain and for pure homogeneous Dirichlet condition.
} 
4, the analysis of the $V^{\mathrm{LTZ}, \mathrm{bl} \pm}-\mathbb{P}_{1}$ pair is completed. Numerical examples are reported in Section 5. Finally in Section 6, some conclusion and comments are given.

\section{Model PROBLEM AND THE FINITE ELEMENT DISCRETISATION}

2.1. The model problem. Let $\Omega$ be a polygonal domain, with the boundary $\Gamma=\partial \Omega$. Moreover, let $\partial \Omega=\Gamma_{D} \cup \Gamma_{N}$, such that $\left|\Gamma_{D}\right|>0$, and $\stackrel{\circ}{\Gamma}_{D} \cap \stackrel{\circ}{\Gamma}_{N}=\emptyset$.

Incompressible Stokes problem. Let $\underset{\sim}{u}$ be the velocity and $p$ be the pressure of a flow. The governing equation is

$$
-2 \mu \operatorname{div} \boldsymbol{\varepsilon}(\underset{\sim}{u})+\nabla p=\underset{\sim}{f}, \quad \operatorname{div} \underset{\sim}{u}=0
$$

where $\boldsymbol{\varepsilon}(\underset{\sim}{u})=\frac{1}{2}\left(\nabla \underset{\sim}{u}+\nabla{\underset{\sim}{u}}^{\top}\right)$. Here $\mu$ is the viscosity. In the sequel, we set $\mu=1$. The mixed boundary condition is imposed as

$$
\underset{\sim}{u}=\underset{\sim}{0} \text { on } \Gamma_{D} \text {, and }(2 \underset{\boldsymbol{\varepsilon}}{(\underset{\sim}{u})}-p \underset{\sim}{\mathbf{I d}}) \cdot \mathbf{n}=0 \text { on } \Gamma_{N} .
$$

Define $\boldsymbol{H}_{D}^{1}(\Omega):=\left\{\underset{\sim}{v} \in\left(H^{1}(\Omega)\right)^{2}: \underset{v}{v}=\underset{\sim}{0}\right.$ on $\left.\Gamma_{D}\right\}$, and adjointly $\boldsymbol{H}_{N}^{1}(\Omega):=\left\{\underset{\sim}{v} \in\left(H^{1}(\Omega)\right)^{2}:\right.$ $\underset{\sim}{\nu}=\underset{\sim}{0}$ on $\left.\Gamma_{N}\right\}$. Denote $L_{*}^{2}(\Omega):=\operatorname{div} \boldsymbol{H}_{D}^{1}(\Omega)$. If $\left|\Gamma_{N}\right|=0, L_{*}^{2}(\Omega)=L_{0}^{2}(\Omega)$, otherwise $L_{*}^{2}(\Omega)=L^{2}(\Omega)$. Then the variational formulation for the incompressible Stokes equation is to find $\underset{\sim}{u}, p) \in \boldsymbol{H}_{D}^{1}(\Omega) \times$ $L_{*}^{2}(\Omega)$, such that

$$
\left\{\begin{aligned}
2(\boldsymbol{\varepsilon}(\underset{\sim}{u}), \boldsymbol{\varepsilon}(\underset{\sim}{v}))+(p, \operatorname{div} \underset{\sim}{\mathcal{v}}) & =(\underset{\sim}{f}, \underset{\sim}{v}) & & \forall \underset{\sim}{v} \in \boldsymbol{H}_{D}^{1}(\Omega) \\
(q, \operatorname{div} \underset{\sim}{u}) & =0 & & \forall q \in L_{*}^{2}(\Omega) .
\end{aligned}\right.
$$

There hold these regularity results for Sobolev spaces, which are crucial for the Babuska-Brezzi conditions associated with the model problem.

Lemma 1. The regularity description of the function spaces below.

(1) (Korn's inequality, [60]) There exists a constant $C_{D}$ such that

$$
\|\nabla \underset{\sim}{w}\|_{0, \Omega} \leqslant C_{D}\|\boldsymbol{\varepsilon}(\underset{\sim}{w})\|_{0, \Omega}, \quad \forall \underset{\sim}{w} \in \boldsymbol{H}_{D}^{1}(\Omega) .
$$

(2) (inf-sup condition, [17, 40, 71]) There exists a constant $C$, such that

$$
\inf _{q \in L_{*}^{2}(\Omega)} \sup _{\underset{\sim}{\mathcal{N} \in \boldsymbol{H}_{D}^{1}(\Omega)}} \frac{(q, \operatorname{div} \underset{\sim}{\sim})}{\|q\|_{0, \Omega}\|\|_{\sim} \|_{1, \Omega}} \geqslant C .
$$


Lemma 2. [19] Given $\underset{\sim}{f} \in\left(\boldsymbol{H}_{D}^{1}(\Omega)\right)^{\prime}$, there exists a unique $\left.\underset{\sim}{u}, p\right) \in \boldsymbol{H}_{D}^{1}(\Omega) \times L_{*}^{2}(\Omega)$, such that (3) holds true. Moreover,

$$
\|\underset{\sim}{u}\|_{1, \Omega}+\|p\|_{0, \Omega} \equiv\|\underset{\sim}{\sim}\|_{\left(\boldsymbol{H}_{D}^{1}(\Omega)\right)^{\prime}}
$$

If $\Omega$ is convex, $\Gamma=\Gamma_{D}$ and further $f \in L^{2}(\Omega)$, then with $C$ a generic constant,

$$
\|\underset{\sim}{u}\|_{2, \Omega}+\|p\|_{1, \Omega} \leqslant C\|f\|_{0, \Omega}
$$

From this point onwards, $\lesssim, \gtrsim$, and $\equiv$ respectively denote $\leqslant, \geqslant$, and $=$ up to a constant. The hidden constants depend on the domain, and, when triangulation is involved, they also depend on the shape-regularity of the triangulation, but they do not depend on $h$ or any other mesh parameter. Nearly incompressible linear elasticity problem. Denote by $\underset{\sim}{u}$ the displacement of an elastic body. The governing equation is

$$
\operatorname{div}(2 \mu \underset{\sim}{\boldsymbol{\varepsilon}} \underset{\sim}{u})+\lambda \operatorname{tr}(\boldsymbol{\varepsilon}(\underset{\sim}{u})) \mathbf{I} \mathbf{d})=\underset{\sim}{f} .
$$

Here $\mu$ is the shear modulus, and $\lambda=\frac{v}{1-2 v}$, where $v$ is the Poisson ratio. Again, in the sequel, we set $\mu=1$, and will mainly focus on the case that $\lambda \gg 1$. We impose the mixed boundary condition

$$
\left.\underset{\sim}{u}=\underset{\sim}{0} \text { on } \Gamma_{D} \text {, and }(2 \underset{\sim}{\boldsymbol{\varepsilon}} \underset{\sim}{u})+\lambda \operatorname{tr}(\boldsymbol{\varepsilon}(\underset{\sim}{u})) \underset{\sim}{\mathbf{I d}}\right) \cdot \mathbf{n}=0 \text { on } \Gamma_{N} .
$$

The variational formulation for the linear elasticity problem is to find $\underset{\sim}{u^{\lambda}} \in \boldsymbol{H}_{D}^{1}(\Omega)$, such that

$$
2\left(\boldsymbol{\varepsilon}\left({\underset{\sim}{u}}^{\lambda}\right), \boldsymbol{\varepsilon}(\underset{\sim}{v})\right)+\lambda\left(\operatorname{div}{\underset{\sim}{u}}^{\lambda}, \operatorname{div} \underset{\sim}{v}\right)=(\underset{\sim}{f}, \underset{\sim}{v}), \quad \forall \underset{\sim}{v} \in \boldsymbol{H}_{D}^{1}(\Omega)
$$

or equivalently, with $p^{\lambda}=\lambda \operatorname{div}{\underset{\sim}{u}}^{\lambda}$, to find $\left({\underset{\sim}{u}}^{\lambda}, p^{\lambda}\right) \in \boldsymbol{H}_{D}^{1}(\Omega) \times L^{2}(\Omega)$, such that

$$
\left\{\begin{aligned}
2 \mu\left(\boldsymbol{\varepsilon}\left({\underset{\sim}{u}}^{\lambda}\right), \boldsymbol{\varepsilon}(\underset{\sim}{v})\right)+\left(p^{\lambda}, \operatorname{div} \underset{\sim}{v}\right) & =(\underset{\sim}{f}, \underset{\sim}{v}), & \forall \underset{\sim}{v} \in \boldsymbol{H}_{D}^{1}(\Omega), \\
\left(\operatorname{div}_{\sim}^{u_{\sim}^{\lambda}}, q\right)-\frac{1}{\lambda}\left(p^{\lambda}, q\right) & =0, & \forall q \in L^{2}(\Omega) .
\end{aligned}\right.
$$

The following estimate follows from the Babuska-Brezzi theory, and Lemma 1.

Lemma 3. [19]

(1) Given $\underset{\sim}{f} \in\left(\boldsymbol{H}_{D}^{1}(\Omega)\right)^{\prime}$, there exists a unique $\left(\underset{\sim}{u^{\lambda}}, p^{\lambda}\right) \in \boldsymbol{H}_{D}^{1}(\Omega) \times L_{*}^{2}(\Omega)$, such that $(9)$ holds true. Moreover,

$$
\left\|\underline{\sim}^{\lambda}\right\|_{1, \Omega}+\left\|p^{\lambda}\right\|_{0, \Omega} \equiv\|f\|_{\left(\boldsymbol{H}_{D}^{1}(\Omega)\right)^{\prime}}
$$


The equivalence does not depend on $\lambda$. If $\Omega$ is convex, $\Gamma=\Gamma_{D}$ and further $\underset{\sim}{f} \in \boldsymbol{L}^{2}(\Omega)$, then

$$
\left\|{\underset{\sim}{u}}^{\lambda}\right\|_{2, \Omega}+\left\|p^{\lambda}\right\|_{1, \Omega} \leqslant C\|f\|_{0, \Omega}
$$

Again, $C$ does not depend on $\lambda$.

(2) Given $\underset{\sim}{f} \in\left(\boldsymbol{H}_{D}^{1}(\Omega)\right)^{\prime}$, there exists a unique $\underset{\sim}{u^{\lambda}} \in \boldsymbol{H}_{D}^{1}(\Omega)$, such that (8) holds true. Moreover,

$$
\left\|{\underset{\sim}{u}}^{\lambda}\right\|_{1, \Omega}+\lambda\left\|\operatorname{div} \underset{\sim}{u^{\lambda}}\right\|_{0, \Omega} \equiv\left\|\sim_{\sim}^{f}\right\|_{\left(\boldsymbol{H}_{D}^{1}(\Omega)\right)^{\prime}}
$$

The equivalence does not depend on $\lambda$. If $\Omega$ is convex, $\Gamma=\Gamma_{D}$ and further $f \in \boldsymbol{L}^{2}(\Omega)$, then

$$
\left\|\underset{\sim}{u^{\lambda}}\right\|_{2, \Omega}+\lambda\|\operatorname{div} \underset{\sim}{u}\|_{1, \Omega} \leqslant C\|f\|_{0, \Omega} .
$$

Again, $C$ does not depend on $\lambda$.

2.2. Finite element discretisation of the model problems. When the Sobolev spaces $\boldsymbol{H}_{D}^{1}(\Omega)$ and $L_{*}^{2}(\Omega)$ are replaced by some finite element spaces $V_{h D}$ and $W_{h}$, conforming or nonconforming, we get the finite element discretisation for Stokes problem (3): find $\left.\underset{\sim}{u_{h}}, p_{h}\right) \in \boldsymbol{V}_{h D} \times W_{h}$, such that

$$
\left\{\begin{aligned}
\left(\boldsymbol{\varepsilon}_{h}\left(\underset{\sim}{u_{h}}\right), \boldsymbol{\varepsilon}_{h}\left(\underline{\sim}_{h}\right)\right)+\left(\operatorname{div}_{h} \underline{\sim}_{h}, p_{h}\right) & =\left(\underset{\sim}{f},{\underset{\sim}{v} h}_{h}\right) & \forall{\underset{\sim}{v} h}_{h} \in \boldsymbol{V}_{h D}, \\
\left(\operatorname{div}_{h}{\underset{\sim}{h}}_{h}, q_{h}\right) & =0 & \forall q_{h} \in W_{h} .
\end{aligned}\right.
$$

Here $\varepsilon_{h}$ and $\operatorname{div}_{h}$ are in the piecewise sense for $V_{h D} \not \subset H_{D}^{1}(\Omega)$.

Simultaneously, we have the finite element discretisation of the linear elasticity problem (9): find $\left({\underset{\sim}{h}}_{h}^{\lambda}, p_{h}^{\lambda}\right) \in \boldsymbol{V}_{h D} \times W_{h}$, such that

$$
\left\{\begin{array}{rll}
\left(\boldsymbol{\varepsilon}_{h}\left(\underline{\sim}_{h}^{\lambda}\right), \boldsymbol{\varepsilon}_{h}\left(\underline{\sim}_{h}\right)\right)+\left(\operatorname{div}_{h}{\underset{\sim}{h}}_{h}, p_{h}^{\lambda}\right) & =\left(\mathbf{f},{\underset{\sim}{v}}_{h}\right) & \forall{\underset{\sim}{v}}_{h} \in \boldsymbol{V}_{h D}, \\
\left(\operatorname{div}_{h}{\underset{\sim}{u}}_{h}^{\lambda}, q_{h}\right)-\frac{1}{\lambda}\left(p_{h}^{\lambda}, q_{h}\right) & =0 & \forall q_{h} \in W_{h} .
\end{array}\right.
$$

We define three stable conditions for the finite element scheme. The subscript " $h$ " in the norms implies the triangulation dependence.

SC 1: There exists a positive constant $\gamma_{1}$, such that $\gamma_{h}^{1}:=\inf _{0 \neq q_{h} \in W_{h}} \sup _{\mathbf{0} \neq \mathcal{\sim}_{h} \in V_{h D}} \frac{\left(\operatorname{div}_{h} \mathcal{V}_{h}, q_{h}\right)}{\left\|\mathcal{v}_{h}\right\|_{1, h}\left\|q_{h}\right\|_{0, \Omega}}>\gamma_{1}$. 
SC 2: The discrete Korn's inequality holds. Namely, there exists a constant $\gamma_{2}$, such that it holds uniformly for the finite element spaces that

$$
\left\|\nabla_{h} \underline{\sim}_{h}\right\|_{0, \Omega} \leqslant \gamma_{2}\left\|\boldsymbol{\varepsilon}_{h}\left(\underline{\sim}_{h}\right)\right\|_{0, \Omega}, \quad \forall \underline{\sim}_{h} \in \boldsymbol{V}_{h D} .
$$

SC 3: $W_{h}=\left\{\operatorname{div}_{h}{\underset{\sim}{h}}_{h}:{\underset{\sim}{h}}_{h} \in \boldsymbol{V}_{h D}\right\}$.

Evidently, the condition SC 3 implies that $\mathbf{Z}_{h}:=\left\{{\underset{\sim}{v}}_{h} \in \boldsymbol{V}_{h D}:\left(\operatorname{div}_{h} \underline{\sim}_{h}, q_{h}\right)=0, \forall q_{h} \in W_{h}\right\}=\left\{{\underset{\sim}{\nu}}_{h} \in\right.$ $\left.\boldsymbol{V}_{h D}: \operatorname{div}_{h} \underline{\sim}_{h}=0\right\}$; namely, SC 3 ensures the divergence-free conditions in a primal way.

The stability conditions SC 1 and SC 2 ensures the validity of the Strang lemma below.

Lemma 4. [22] Let $\boldsymbol{V}_{h D}$ and $W_{h}$ be a finite element pair that the conditions $\boldsymbol{S C} 1$ and $\mathbf{S C} 2$ hold.

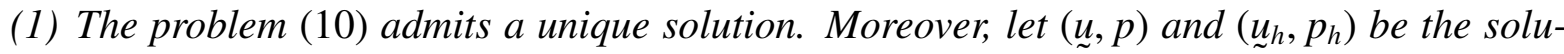
tions of (3) and (10), respectively. Then

$$
\begin{aligned}
& \left\|\underset{\sim}{u}-{\underset{\sim}{u}}_{h}\right\|_{1, h}+\left\|p-p_{h}\right\|_{0, \Omega} \leqslant C\left(\inf _{\underline{v}_{h} \in V_{h D}}\left\|\underset{\sim}{u}-\underline{\sim}_{h}\right\|_{1, h}+\inf _{q_{h} \in W_{h}}\left\|p-q_{h}\right\|_{0, \Omega}\right. \\
& \left.+\sup _{0 \neq \mathcal{V}_{h} \in \boldsymbol{V}_{h D}} \frac{\left(\boldsymbol{\varepsilon}_{h}(\underset{\sim}{u}), \boldsymbol{\varepsilon}_{h}\left(\underline{\sim}_{h}\right)\right)+\left(\operatorname{div}_{h} \underline{\sim}_{h}, p\right)-\left({\underset{\sim}{f}}_{\sim} v_{h}\right)}{\left\|\underline{v}_{h}\right\|_{1, h}}\right) .
\end{aligned}
$$

(2) The problem (11) admits a unique solution. Moreover, let $\left(\underset{\sim}{u^{\lambda}}, p^{\lambda}\right)$ and $\left(\underset{\sim}{u_{h}^{\lambda}}, p_{h}^{\lambda}\right)$ be the solutions of (9) and (11), respectively. Then, with $C$ a constant independent of $\lambda$,

$$
\begin{aligned}
& \left\|\underline{\sim}^{\lambda}-\underline{u}_{h}^{\lambda}\right\|_{1, h}+\left\|p^{\lambda}-p_{h}^{\lambda}\right\|_{0, \Omega} \leqslant C\left(\inf _{\underline{v}_{h} \in V_{h D}}\left\|{\underset{\sim}{\sim}}^{\lambda}-{\underset{\sim}{h}}_{h}\right\|_{1, h}+\inf _{q_{h} \in W_{h}}\left\|p^{\lambda}-q_{h}\right\|_{0, \Omega}\right.
\end{aligned}
$$

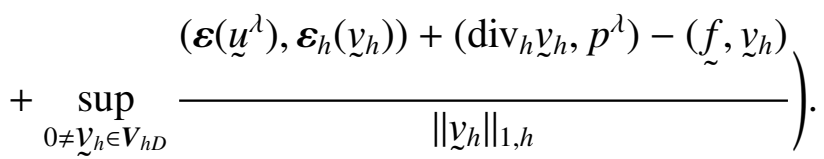

For the finite element pairs for which SC 1, SC 2 and SC 3 all hold, we consider the primal

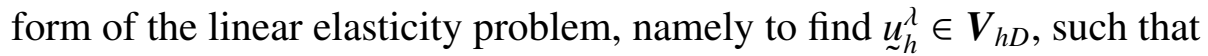

$$
\left(\boldsymbol{\varepsilon}_{h}\left({\underset{\sim}{h}}_{h}^{\lambda}\right), \boldsymbol{\varepsilon}_{h}\left(\underline{\sim}_{h}\right)\right)+\lambda\left(\operatorname{div}{\underset{\sim}{u}}_{h}^{\lambda}, \operatorname{div}{\underset{\sim}{v} h}_{h}\right)=\left(\underset{\sim}{f},{\underset{\sim}{v}}_{h}\right), \quad \forall{\underset{\sim}{v}}_{h} \in \boldsymbol{V}_{h D}
$$

The solution of (13) is equal to the displacement of the solution of (11), and we have similar analysis immediately. 


\subsection{Finite element spaces.}

2.3.1. Subdivision of the domain. Let $\Omega$ be a polygonal domain. For simplicity, we assume it can be subdivided to a rectangular grid $\mathcal{G}_{h}$. For the grid $\mathcal{G}_{h}$, let $\mathcal{N}_{h}$ denote the set of all the vertices, $\mathcal{N}_{h}=\mathcal{N}_{h}^{i} \cup \mathcal{N}_{h}^{b}$, with $\mathcal{N}_{h}^{i}$ and $\mathcal{N}_{h}^{b}$ consisting of the interior vertices and the boundary vertices, respectively. Similarly, let $\mathcal{E}_{h}=\mathcal{E}_{h}^{i} \cup \mathcal{E}_{h}^{b}$ denote the set of all the edges, with $\mathcal{E}_{h}^{i}$ and $\mathcal{E}_{h}^{b}$ consisting of the interior edges and the boundary edges, respectively. Associated with the boundary, denote by $\mathcal{E}_{h}^{D}$ and $\mathcal{E}_{h}^{N}$ the set of edges on $\Gamma_{D}$ and $\Gamma_{N}$, respectively. Then $\mathcal{E}_{h}^{b}=\mathcal{E}_{h}^{D} \cup \mathcal{E}_{h}^{N}$. For an edge $e, \mathbf{n}_{e}$ is a unit vector normal to $e$, and $\tau_{e}$ is a unit tangential vector of $e$ such that $\mathbf{n}_{e} \times \tau_{e}>0$. For $e \in \mathcal{E}_{h}^{i}$, $K_{e}^{L}$ and $K_{e}^{R}$ are the two adjacent elements that share $e$ as the common edge, and $\mathbf{n}_{e}^{L}$ and $\mathbf{n}_{e}^{R}$ denote the unit outer normal vectors of $K_{e}^{L}$ and $K_{e}^{R}$, respectively, on $e$. On the edge $e$, we use $\llbracket \cdot \rrbracket_{e}$ for the jump across $e$. Namely, for $v$ that makes the definition below make sense,

$$
\llbracket v \rrbracket= \begin{cases}\left.v\right|_{T_{e}^{L}}\left(\mathbf{n}_{e} \cdot \mathbf{n}_{e}^{L}\right)+\left.v\right|_{T_{e}^{R}}\left(\mathbf{n}_{e} \cdot \mathbf{n}_{e}^{R}\right), & e \in \mathcal{E}_{h}^{i}, \\ \left.v\right|_{K} & e \in \mathcal{E}_{h}^{b}, e \subset \partial K .\end{cases}
$$

Assumption AG. Associated with the boundary conditions, we assume the grid $\mathcal{G}_{h}$ satisfies the assumptions below:

(1) every point in $\overline{\Gamma_{D}} \cap \overline{\Gamma_{N}}$ is a vertex of the grid,

(2) at every segment of $\partial \Omega$, every connected part of $\Gamma_{D}$ and $\Gamma_{N}$ each has at least one interior vertex, namely a vertex which is not in $\overline{\Gamma_{D}} \cap \overline{\Gamma_{N}}$ nor a corner vertex.

(3) every cell has at least one vertex in the interior of the domain.

(4) every interior edge, namely an edge shared by two cells, has at least one interior vertex as its end.

In the sequel, for simplicity, we assume that both $\Gamma_{D}$ and $\Gamma_{N}$ are connected.

It is obvious that an interior vertex is shared by four cells. An interior vertex is shared by four interior edges. For rectangular grids, a macroelement is defined as the combination of four cells that share a same vertex. See Figure 1 for an illustration.

Obviously every interior vertex $\underset{\sim}{x}$ of $\mathcal{G}_{h}$ is the interior vertex of the macroelement composing of the cells that share $\underset{\sim}{x}$ as a common vertex. Therefore, every cell, as it has at least one interior vertex, is covered by at least one macroelement. Simultaneously, every cell is covered by no more than four macroelements.

Lemma 5. Let $\underset{\sim}{x}$ be a boundary vertex which is not a corner. Then there exists at least one macroelement $M$, such that $\underset{\sim}{x}$ is a boundary vertex but a corner of $M$. 


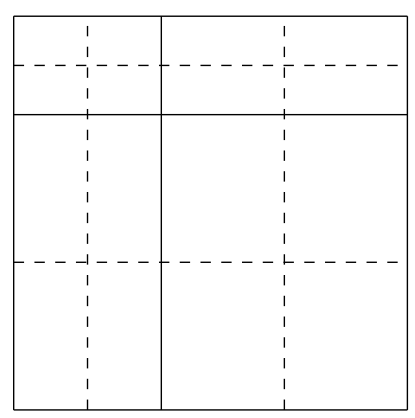

(a macroelement)

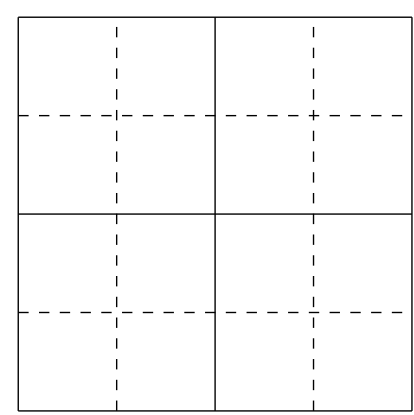

(a reference macroelement)

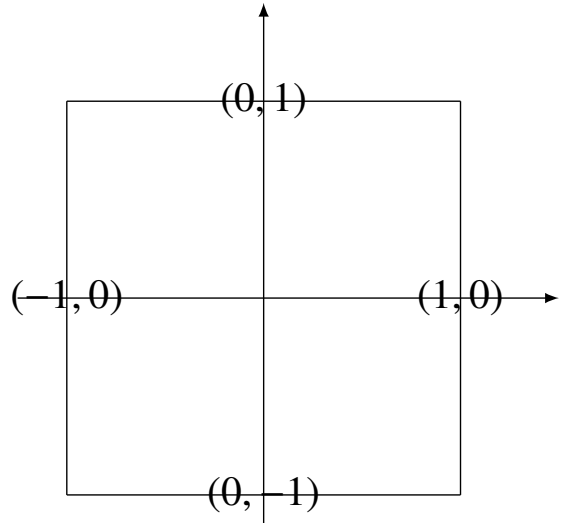

(a reference rectangle)

FIGURE 1. Illustration of macroelements

If a macroelement $M$ has an edge, which is the combination of two neighboured edges of $\mathcal{G}_{h}$, contained in $\Gamma_{N}$, then this macroelement $M$ is called to be of type $\mathbf{N}$. Denote $\mathcal{M}_{h}$ as the set of macroelements in $\mathcal{G}_{h}$. The lemma below is straightforward.

Lemma 6. Assume AG hold, then

(1) given $K \in \mathcal{G}_{h}$, there is an $M \in \mathcal{M}_{h}$, such that $K \subset M$; moreover, $K$ is covered by no more than 4 macroelements;

(2) given an interior edge e, there is an $M \in \mathcal{M}_{h}$, such that e is an interior edge of $M$;

(3) given an edge $e \in \mathcal{E}_{h}^{N}$, there is an $M \in \mathcal{M}_{h}$ of type $N$, such that e $\subset \partial M$.

In the sequel, we will always assume the assumptions AG hold, without special declaration.

2.3.2. Several finite element spaces. For a rectangle $R$, denote by $P_{k}(R)$ the space of $k^{\text {th }}$-degree polynomials, and $Q_{k}(R)$ the bi- $k^{\text {th }}$-degree polynomial space. Denote $0 \not \equiv \phi_{R}^{b} \in Q_{2}(R)$ such that $\phi_{R}^{b}$ vanishes on the boundary of $R$, and $0 \not \equiv \phi_{R}^{\mathrm{n}} \in P_{2}(R)$ such that $\int_{e} \phi_{R}^{\mathrm{n}} q$ vanishes on every edge $e$ of $R$, and for every $q \in P_{1}(e)$. With respect to these notation, denote $Q_{1}^{+}(R)=Q_{1}(R)+\operatorname{span}\left\{\phi_{R}^{b}\right\}$, and $Q_{1}^{ \pm}(R)=Q_{1}(R)+\operatorname{span}\left\{\phi_{R}^{\mathrm{n}}\right\}$. We also denote by $Q^{\mathrm{LTZ}}(R)$ the shape function space of LinTobiska-Zhou element. Particularly, on the rectangle $R=\left(x_{c}-\xi, x_{c}+\xi\right) \times\left(y_{c}-\eta, y_{c}+\eta\right), \phi_{R}^{b}=$ $\left(\left(x-x_{c}\right)^{2}-\xi^{2}\right)\left(\left(y-y_{c}\right)^{2}-\eta^{2}\right), \phi_{R}^{\mathrm{n}}=3 \eta^{2}\left(x-x_{c}\right)^{3}+3 \xi^{2}\left(y-y_{c}\right)^{2}-4 \xi^{2} \eta^{2}$, and $Q^{\mathrm{LTZ}}=\operatorname{span}\left\{1, x, y, x^{2}, y^{2}\right\}$.

We define several finite element spaces as below. Define

$$
\begin{gathered}
V_{h}^{\mathrm{bq}}:=\left\{v_{h} \in H^{1}(\Omega):\left.v_{h}\right|_{K} \in Q_{2}(K), \forall K \in \mathcal{G}_{h}\right\}, \\
V_{h}^{\mathrm{bl}+}:=\left\{v_{h} \in H^{1}(\Omega):\left.v_{h}\right|_{K} \in Q_{1}^{+}(K), \forall K \in \mathcal{G}_{h}\right\},
\end{gathered}
$$




$$
\begin{aligned}
& V_{h}^{\mathrm{bl} \pm}:=\left\{v_{h} \in L^{2}(\Omega):\left.v_{h}\right|_{K} \in Q_{1}^{ \pm}(K), \forall K \in \mathcal{G}_{h},\right. \\
& \left.\qquad \int_{e} v_{h} q_{e} \text { is continuous across internal edge } e, \forall q_{e} \in P_{1}(e)\right\}, \\
& V_{h}^{\mathrm{LTZ}}:=\left\{v_{h} \in L^{2}(\Omega):\left.v_{h}\right|_{K} \in Q^{\mathrm{LTZ}}(K), \forall K \in \mathcal{G}_{h}, \int_{e} v_{h} \text { is continuous across internal edge } e\right\},
\end{aligned}
$$

and

$$
\mathbb{P}_{1}:=\left\{q_{h} \in L^{2}(\Omega):\left.q_{h}\right|_{K} \in P_{1}(K) \forall K \in \mathcal{G}_{h}\right\} .
$$

Evidently, $V_{h}^{\mathrm{bl} \pm}=V_{h}^{\mathrm{bl}} \oplus \operatorname{span}\left\{\phi_{R}^{\mathrm{n}}\right\}_{R \in \mathcal{G}_{h}}$, where $V_{h}^{\mathrm{bl}}$ is the standard bilinear element space.

Remark 7. To construct the spaces $V_{h}^{\mathrm{bl}+}$ and $V_{h}^{\mathrm{bl} \pm}$, we will construct the bilinear element space $V_{h}^{\mathrm{bl}}$ and a bubble function space for each. The space $V_{h}^{\mathrm{bl}+}$ consists of $V_{h}^{\mathrm{bl}}$ and a space of bubble functions, namely one $\phi_{R}^{b}$ on each cell; the space $V_{h}^{\mathrm{bl} \pm}$ consists of $V_{h}^{\mathrm{bl}}$ and another space of bubble functions, namely one $\phi_{R}^{n}$ on each cell.

2.4. The main results of this paper. Define associated with the boundary condition

$$
V_{h D}^{\mathrm{LTZ}}=\left\{v_{h} \in V_{h}^{\mathrm{LTZ}}: \int_{e} v_{h}=0 \forall e \in \mathcal{E}_{h}^{D}\right\},
$$

and

$$
V_{h D}^{\mathrm{bl} \pm}=\left\{v_{h} \in V_{h}^{\mathrm{bl} \pm}: \int_{e} v_{h} \delta_{h}=0 \forall \delta_{h} \in P_{1}(e), \forall e \in \mathcal{E}_{h}^{D}\right\},
$$

and $\boldsymbol{V}_{h D}^{\mathrm{LTZ}, \mathrm{bl} \pm}:=V_{h D}^{\mathrm{LTZ}} \times V_{h D}^{\mathrm{bl} \pm}$. Adjointly, $\boldsymbol{V}_{h N}^{\mathrm{LTZ}, \mathrm{bl} \pm}$ is defined the same way. Then $\boldsymbol{V}_{h D}^{\mathrm{LTZ}, \mathrm{bl} \pm}-\mathbb{P}_{1}$ forms a nonconforming pair for the boundary value problems.

We substitute $\boldsymbol{V}_{h D}^{\mathrm{LTZ}, \mathrm{bl} \pm}$ in the finite element element problems defined in Section 2.2 to take the place of $\boldsymbol{V}_{h D}$, and $\mathbb{P}_{1}$ for the role of $W_{h}$. The $\boldsymbol{V}_{h D}^{\mathrm{LTZ}, \mathrm{bl} \pm}-\mathbb{P}_{1}$ pair satisfies the conditions SC 1, SC 2 and SC 3, and it provides a convergent discretisation scheme for the model problems. Below are the main results of the paper. We postpone the proof of SC 1, SC 2 and SC 3 to Section 4.

\subsubsection{Discretisation of the Stokes problem.}

Theorem 8. Let $(\underset{\sim}{u}, p)$ and $\left(\underset{\sim}{u_{h}}, p_{h}\right)$ be the solutions of (3) and (10), respectively. Then $\operatorname{div}_{h} \underset{\sim}{u_{h}}=0$.

Moreover,

(1) if $\underset{\sim}{u} \in\left(H^{2}(\Omega)\right)^{2} \cap \boldsymbol{H}_{D}^{1}(\Omega)$ and $p \in H^{1}(\Omega)$, then

$$
\|\underset{\sim}{u}-\underset{\sim}{u}\|_{1, h}+\left\|p-p_{h}\right\|_{0, \Omega} \leqslant C h\left(|\underset{\sim}{u}|_{2, \Omega}+|p|_{1, \Omega}\right) ;
$$


(2) if $\Omega$ is convex and $\Gamma_{D}=\partial \Omega$, then $\|\underset{\sim}{u}-\underset{\sim}{u}\|_{0, \Omega} \leqslant C h^{2}\|f\|_{0, \Omega}$.

Proof. For the energy norm estimate, by Lemma 4, we have to estimate the approximation error and the consistent error separately. As the finite element space contain complete linear polynomials, by Lemma 4, we only have to estimate the consistency term

$$
\begin{aligned}
& \left(\boldsymbol{\varepsilon}_{h}(\underset{\sim}{u}), \boldsymbol{\varepsilon}_{h}\left({\underset{\sim}{h}}_{h}\right)\right)+\left(\operatorname{div}_{h} \underline{\sim}_{h}, p\right)-\left(\underset{\sim}{f},{\underset{\sim}{h}}_{h}\right) \\
& \sup _{0 \neq \mathcal{V}_{h} \in V_{h D}} \longrightarrow\left\|\underline{\nu}_{\sim}\right\|_{1, h}
\end{aligned}
$$

The estimate falls into the category of standard techniques; c.f., e.g., [55, 65, 72]. Actually, since $\underset{\sim}{u} \in\left(H^{2}(\Omega)\right)^{2}$ and $p \in H^{1}(\Omega)$, we have

$$
\left(\boldsymbol{\varepsilon}_{h}(\underset{\sim}{u}), \boldsymbol{\varepsilon}_{h}\left({\underset{\sim}{h}}_{h}\right)\right)+\left(\operatorname{div}_{h}{\underset{\sim}{h}}_{h}, p\right)-\left(\underset{\sim}{f},{\underset{\sim}{v} h}_{h}\right)=\left.\sum_{K \in \mathcal{T}_{h}} \int_{\partial K} \underset{\sim}{v_{h}^{1}}\right|_{K} \sigma_{K}^{1}+\left.{\underset{\sim}{\nu}}_{h}^{2}\right|_{K} \sigma_{K}^{2} d s
$$

where $\sigma_{K}^{1}=\left(\left.2 \boldsymbol{\varepsilon}(\underset{\sim}{u}) \cdot \mathbf{n}\right|_{K}\right)_{x}-p \mathbf{n}_{x}$ and $\sigma_{K}^{2}=\left(\left.2 \boldsymbol{\varepsilon}(\underset{\sim}{u}) \cdot \mathbf{n}\right|_{K}\right)_{y}-p \mathbf{n}_{y}$. Note that $\int_{e} \llbracket{\underset{\sim}{~}}_{h}^{2} \rrbracket q_{e}=0$ for any $q_{e} \in P_{1}(e)$ and any edge $e$, we obtain by standard argument that

$$
\left.\sum_{K \in \mathcal{T}_{h}} \int_{\partial K} \underset{\sim}{v_{h}^{2}}\right|_{K} \sigma^{2} \leqslant C h^{k}|| \sigma^{2} \|_{k, \Omega}\left|v_{\sim}^{2}\right|_{1, h} \text { for } \sigma^{2} \in H^{k}(\Omega), \quad k=1,2 .
$$

Since ${\underset{\sim}{\nu}}_{h}^{1} \in V_{h}^{\mathrm{LTZ}}$, direct computation leads to that (Lemma 3 of [52]) $\sum_{e \subset \partial K} \int_{e}\left(\underline{\sim}_{h}^{1}-f_{e} v_{h}^{1}\right) q_{K}=0$ for $q_{K} \in P_{1}(K)$ on any cell $K$. Noting that $\int_{e} \llbracket v_{\sim}^{1} \rrbracket q_{e}=0$ for any $q_{e} \in P_{0}(e)$ on any edge $e$, we have

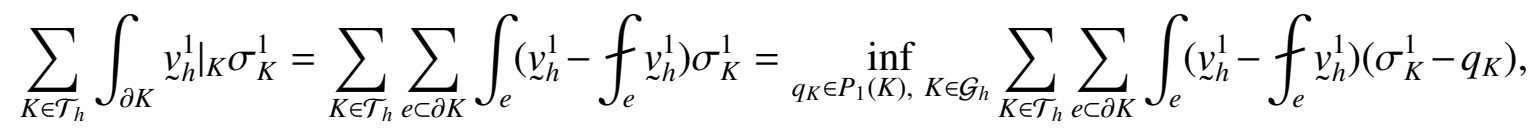

and thus

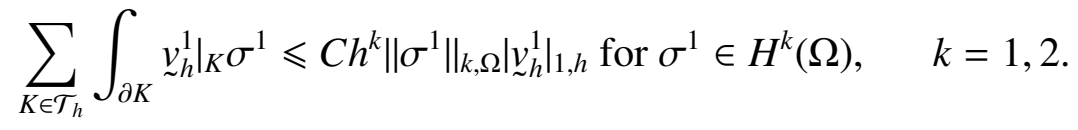

Therefore the estimate (14) follows. The $L^{2}$ error estimate follows by a standard duality argument. Concerning the nonconforming essence of the finite element, we refer to Theorem 10.3.21 of [18] for some technical details. The proof is finished.

2.4.2. Discretisation of the linear elasticity problem. For the discretisation of the linear elasticity problem, we mainly have the same results.

Theorem 9. Let $\left(\underset{\sim}{u^{\lambda}}, p^{\lambda}\right)$ and $\left(\underset{\sim}{u_{h}^{\lambda}}, p_{h}^{\lambda}\right)$ be the solutions of (9) and (11), respectively. Then 
(1) if $\underset{\sim}{u^{\lambda}} \in\left(H^{2}(\Omega)\right)^{2} \cap \boldsymbol{H}_{D}^{1}(\Omega)$ and $p^{\lambda} \in H^{1}(\Omega)$, then

$$
\left\|{\underset{\sim}{u}}^{\lambda}-\underline{\sim}_{h}^{\lambda}\right\|_{1, h}+\left\|p^{\lambda}-p_{h}^{\lambda}\right\|_{0, \Omega} \leqslant C h\left(\left|{\underset{\sim}{u}}^{\lambda}\right|_{2, \Omega}+\left|p^{\lambda}\right|_{1, \Omega}\right)
$$

(2) if $\Omega$ is convex and $\Gamma_{D}=\partial \Omega$, then $\left\|{\underset{\sim}{u}}^{\lambda}-{\underset{\sim}{u}}_{h}^{\lambda}\right\|_{0, \Omega} \leqslant C h^{2}\|f\|_{\sim, \Omega}$.

Here $C$ is a generic positive constant that is uniform with respect to $\lambda$.

Theorem 10. Let $\underset{\sim}{u^{\lambda}}$ and ${\underset{\sim}{u}}_{h}^{\lambda}$ be the solutions of (8) and (13), respectively. Then it holds that

(1) if ${\underset{\sim}{u}}^{\lambda} \in\left(H^{2}(\Omega)\right)^{2} \cap \boldsymbol{H}_{D}^{1}(\Omega)$, then

$$
\left\|{\underset{\sim}{u}}^{\lambda}-{\underset{\sim}{u}}_{h}^{\lambda}\right\|_{1, h}+\lambda\left\|\operatorname{div} \underset{\sim}{u^{\lambda}}-\operatorname{div}_{h}{\underset{\sim}{u}}_{h}^{\lambda}\right\|_{0, \Omega} \leqslant C h\left(\left|{\underset{\sim}{u}}^{\lambda}\right|_{2, \Omega}+\lambda\left|\operatorname{div} \underset{\sim}{u^{\lambda}}\right|_{1, \Omega}\right)
$$

(2) if $\Omega$ is convex and $\Gamma_{D}=\partial \Omega$, then $\left\|\underset{\sim}{u^{\lambda}}-{\underset{\sim}{u}}_{h}^{\lambda}\right\|_{0, \Omega} \leqslant C h^{2}\|f\|_{0, \Omega}$.

Here $C$ is a generic positive constant that is uniform with respect to $\lambda$.

The proof of Theorems 9 and 10 are the same as that of Theorem 8 in a standard way, and we omit here. Actually, Theorem 9 can be proved by following the line of the proof of Theorems 2.4 of [53], while the consistency analysis is done the same way as Theorem 8. For Theorem 10, we only have to note that in the two theorems, $p^{\lambda}=\operatorname{div}_{\sim}^{\underset{\sim}{u^{\lambda}}}$, and $p_{h}^{\lambda}=\operatorname{div}_{h} \underset{\sim}{u_{h}^{\lambda}}$. It then remains to us to prove the stability conditions. We will construct the stability conditions in next two Sections.

\section{AN AUXILIARY CONFORMING PAIR AND ITS STABILITY}

For the boundary value problems, we define the vector finite element space:

$$
\boldsymbol{V}_{h}^{\mathrm{bl}+\mathrm{bq}}=V_{h}^{\mathrm{bl}+} \times V_{h}^{\mathrm{bq}},
$$

and associated with the boundary conditions,

$$
\boldsymbol{V}_{h D}^{\mathrm{bl+}, \mathrm{bq}}=\boldsymbol{V}_{h}^{\mathrm{bl}+, \mathrm{bq}} \cap \boldsymbol{H}_{D}^{1}(\Omega), \quad \boldsymbol{V}_{h N}^{\mathrm{bl+}+\mathrm{bq}}=\boldsymbol{V}_{h}^{\mathrm{bl}+, \mathrm{bq}} \cap \boldsymbol{H}_{N}^{1}(\Omega) .
$$

Then $\boldsymbol{V}_{h D}^{\mathrm{bl}+\text {,bq }}-\mathbb{P}_{1}$ forms a conforming pair for the boundary value problems. The main result of this section is the inf-sup condition below.

Theorem 11. Given assumptions $A G$, there exists a constant $C$ depending on $\Omega, \Gamma_{D}, \Gamma_{N}$ and the shape regularity of $\mathcal{G}_{h}$ only, such that

$$
\inf _{q_{h} \in \mathbb{P}_{1}} \sup _{\underline{\sim}_{h} \in V_{h D}^{\mathrm{bl}+\mathrm{bq}}} \frac{\left(q_{h}, \operatorname{div}{\underset{\sim}{h}}_{h}\right)}{\left\|q_{h}\right\|_{0, \Omega}\left\|\underline{\sim}_{h}\right\|_{1, \Omega}} \geqslant C .
$$


We follow the main line of $[53,66]$ and divide the proof of Theorem 11 to several technical lemmas. We begin with the mesh-dependent norm defined on $\mathbb{P}_{1}$ :

$$
\left\|q_{h}\right\|_{h}^{2}=\sum_{K \in \mathcal{G}_{h}} h_{K}^{2}\left\|\nabla q_{h}\right\|_{0, K}^{2}+\sum_{e \in \mathcal{E}_{h}^{i} \cup \mathcal{E}_{h}^{D}} h_{e} \int_{e} \llbracket q_{h} \rrbracket^{2} d s .
$$

Let $M$ be an arbitrary macroelement. Define $Q_{M}$ the space of piecewise linear polynomials on $M, \boldsymbol{V}_{M 0}^{\mathrm{bl}+\mathrm{bq}}=\boldsymbol{V}_{h}^{\mathrm{bl+}, \mathrm{bq}} \cap \boldsymbol{H}_{0}^{1}(M)$, and $\boldsymbol{V}_{M D}^{\mathrm{bl}+\mathrm{bq}}:=\left\{{\underset{\sim}{v}}_{h} \in \boldsymbol{V}_{h D}^{\mathrm{bl}+\text { bq }}:{\underset{\sim}{~}}_{h}\right.$ vanishes in $\left.\Omega \backslash M\right\}$.

On $Q_{M}$ we define a norm and a semi-norm, namely

$$
\left\|q_{h}\right\|_{M}^{2}=\sum_{K \subset M} h_{K}^{2}\left\|\nabla q_{h}\right\|_{0, K}^{2}+\sum_{e \in \mathcal{E}_{M}} h_{e} \int_{e} \llbracket q_{h} \rrbracket^{2} d s
$$

and

$$
\left|q_{h}\right|_{M}^{2}=\sum_{K \subset M} h_{K}^{2}\left\|\nabla q_{h}\right\|_{0, K}^{2}+\sum_{e \in \mathcal{E}_{M}^{i}} h_{e} \int_{e}\left|\llbracket q_{h} \rrbracket\right|^{2} d s,
$$

where $\mathcal{E}_{M}=\left\{e \in \mathcal{E}_{h}: e \subset \bar{M}\right\}$, and $\mathcal{E}_{M}^{i}=\left\{e \in \mathcal{E}_{M}: e \not \subset \partial M\right\}$. Evidently $\|\cdot\|_{M}$ is a norm on $Q_{M}$, and $|\cdot|_{M}$ is a semi-norm on $Q_{M}$ with a kernel of constant functions on $M$.

Lemma 12. There is a positive constant $C$ depending on the shape regularity of the grid such that

$$
\sup _{\mathbf{0} \neq \mathcal{V}_{h} \in \boldsymbol{V}_{M 0}^{\mathrm{bl}+\text { bq }}} \frac{\left(\operatorname{div}_{h} \underset{\sim}{\left.\mathcal{v}_{h}, p_{h}\right)_{M}}\right.}{\left|{\underset{\sim}{h}}_{h}\right|_{1, M}} \geqslant C\left|p_{h}\right|_{M}, \quad p_{h} \in Q_{M},
$$

holds for any $M$.

Proof. First of all, it can be proved that given $q_{h} \in Q_{M}$, such that $\left(\operatorname{div}{\underset{\sim}{~}}_{h}, q_{h}\right)_{M}=0$ for all ${\underset{\sim}{h}}_{h} \in$ $\boldsymbol{V}_{M 0}^{\mathrm{bl}+\text {,bq }}$, then $\left|q_{h}\right|_{M}=0$. Indeed, assume that $\left.q_{h}\right|_{K_{i}}=\alpha_{i}+\beta_{i} \hat{x}_{i}+\gamma_{i} \hat{y}_{i}$, where $\hat{x}_{i}$ and $\hat{y}_{i}$ are the local coordinate on $K_{i}$.(See Figure 2-(A) for an illustration.) Then we can choose $\underset{\sim}{\varphi_{i}} \in v_{M}$, such that

$$
{\underset{\sim}{i}}_{i}= \begin{cases}\left(\beta_{i} \phi_{K_{i}}^{b}, \gamma_{i} \phi_{K_{i}}^{b}\right)^{t} & \text { on } K_{i}\left(\text { see Section 2.3.2 for } \phi_{K_{i}}^{b}\right) \\ 0 & \text { otherwise. }\end{cases}
$$

and it is easy to obtain that $\left(\operatorname{div}{\underset{\sim}{i}}_{i}, q_{h}\right)=\beta_{i}^{2}+\gamma_{i}^{2}$. As $q_{h}$ is assumed such that $\left(\operatorname{div} \underset{\sim}{\varphi_{i}}, q_{h}\right)=0$, we have $\beta_{i}=\gamma_{i}=0, i=1,2,3,4$. Then for any ${\underset{\sim}{h}}_{h}=\left(v_{1}, v_{2}\right)^{\top} \in \boldsymbol{V}_{M 0}^{\mathrm{bl+}, \mathrm{bq}}$, we have, (See Figure 2-(A) 
for an illustration)

$$
\left(\operatorname{div} \underset{\sim}{v_{h}}, q_{h}\right)_{M}=\int_{e_{1}} v_{1}\left(\alpha_{1}-\alpha_{2}\right)+\int_{e_{3}} v_{1}\left(\alpha_{4}-\alpha_{3}\right)+\int_{e_{2}} v_{2}\left(\alpha_{2}-\alpha_{3}\right)+\int_{e_{4}} v_{2}\left(\alpha_{1}-\alpha_{4}\right) .
$$

We choose $v_{2}=0$ and $v_{1}$ being a continuous piecewise bilinear function which is 1 on $C$ and vanishes on other vertices. Then $\int_{e_{1}} v_{1}=\int_{e_{3}} v_{1} \neq 0$. This leads to that $\alpha_{1}-\alpha_{2}+\alpha_{4}-\alpha_{3}=0$. Similarly, we can choose $v_{1}=0$, and $v_{2}$ being a continuous piecewise biquadratic function, so that $\int_{e} v_{2}$ vanishes on all edges $e$ but $e_{2}$. Then we obtain $\alpha_{2}=\alpha_{3}$. Similarly $\alpha_{1}=\alpha_{4}$. Further $\alpha_{1}=\alpha_{2}=\alpha_{3}=\alpha_{4}$; namely $q_{h}$ is a constant function on $M$, and thus $\left|q_{h}\right|_{M}=0$.

$$
(\operatorname{div} \underset{\sim}{v}, q)_{M}
$$

Therefore, for any $M$ given, $\sup _{\mathbf{0} \neq \mathcal{\sim}_{h} \in V_{M 0}^{\mathrm{bl}+\mathrm{bq},|q|_{M} \neq 0}} \frac{}{\left.\left|{\underset{\sim}{\nu} h}_{1, M}\right| q\right|_{M}}>0$. Note that any $M$ can be continuously transformed from a reference macroelement, and $\sup _{\mathbf{0} \neq \mathcal{V}_{h} \in \boldsymbol{V}_{M 0}^{\mathrm{bl+bq},|q|_{M} \neq 0}} \frac{\left(\operatorname{div} \underline{\sim}_{h}, q\right)_{M}}{\left|\underline{\sim}_{h}\right|_{1, M}|q|_{M}}$ varies continu$(\operatorname{div} \underset{\sim}{v}, q)_{M}$

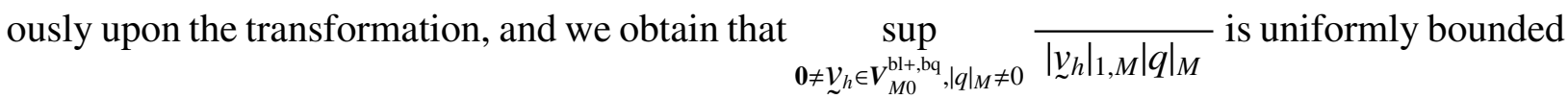
from below for a shape-regular family of macroelements.

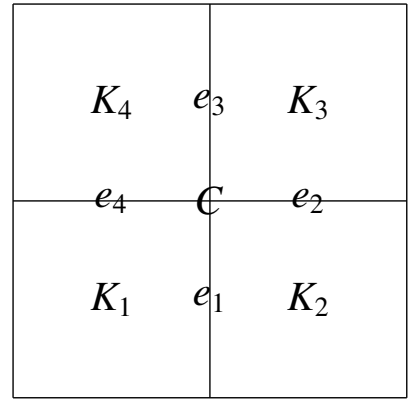

(A)

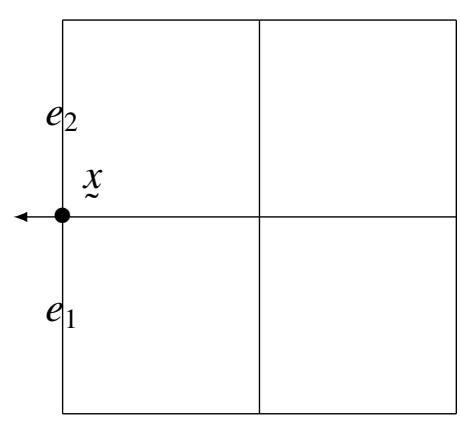

(B)

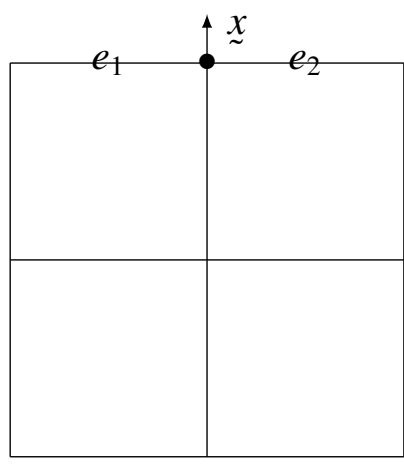

(C)

FIGURE 2. Illustration of macroelements

Lemma 13. There is a positive constant $C$, such that if $M$ has an edge, which is the combination of two neighboured edges of $\mathcal{G}_{h}$, contained in $\Gamma_{N}$, then

$$
\sup _{\mathbf{0} \neq \mathcal{V}_{\sim} \in \boldsymbol{V}_{M D}} \frac{\left(\operatorname{div}_{h} \underset{\sim}{\left.\mathcal{V}_{h}, p_{h}\right)_{M}}\right.}{\left|\underline{\sim}_{h}\right|_{1, M}} \geqslant C\left\|p_{h}\right\|_{M}, \quad p_{h} \in Q_{M} .
$$


Proof. First of all, we show that if $\left(\operatorname{div}{\underset{\sim}{h}}_{h}, q_{h}\right)=0$ for all ${\underset{\sim}{h}}_{h} \in \boldsymbol{V}_{M D}^{\mathrm{bl}+\text {,bq }}$, then $q_{h}=0$. Provided Lemma 12 , this is equivalent to show that, if $q_{h} \not \equiv 0$ is a constant function on $M$, then there exists ${\underset{\sim}{v}}_{h} \in \boldsymbol{V}_{M D}^{\mathrm{bl}+\text {,bq }}$, such that $\left(q_{h}, \operatorname{div}{\underset{\sim}{\nu}}_{h}\right) \neq 0$. It is sufficient to show the existence of such ${\underset{\sim}{v}}_{h}$ that $\int_{M}{\underset{\sim}{v_{h}}}_{h} \neq 0$. Denote ${\underset{\sim}{h}}_{h}=\left(v^{1}, v^{2}\right)$; then

$$
\int_{M} \operatorname{div}{\underset{\sim}{~}}_{h}=\int_{\partial M} \underset{\sim}{v_{h}} \cdot \mathbf{n}=\int_{\partial M} v^{1} \mathbf{n}_{1}+v^{2} \mathbf{n}_{2} .
$$

Let $E$ be an edge of $M$, which is a combination of $e_{1}, e_{2} \in \mathcal{E}_{h}^{N}$, and $\underset{\sim}{x}=\overline{e_{1}} \cap \overline{e_{2}}$.

(1) If $\mathbf{n}_{1}(\underset{\sim}{x}) \neq 0$, (see Figure 2-(B)) then we can choose $v^{1}$ to be the bilinear function such that $v^{1}(\underset{\sim}{x})=1$ and vanishes on other 8 vertices of $M$, and $v^{2}=0$. This way, $\int_{\partial M} v^{1} \mathbf{n}_{1}+v^{2} \mathbf{n}_{2}=$ $\int_{e_{1} \cup e_{2}} v^{1} \mathbf{n}_{1} \neq 0$.

(2) If $\mathbf{n}_{2}(\underset{\sim}{x}) \neq 0$, (see Figure $\left.2-(\mathrm{C})\right)$ then we can choose $v^{1}=0$, and $v^{2}$ to be a piecewise biquadratic function, such that $\int_{e_{1}} v^{2}=\int_{e_{2}} v^{2}=1$, and $\int_{e} v^{2}$ vanishes for all other edge $e$ on $\partial M$. This way, $\int_{\partial M} v^{1} \mathbf{n}_{1}+v^{2} \mathbf{n}_{2}=\int_{e_{1} \cup e_{2}} v^{2} \mathbf{n}_{2} \neq 0$.

The remaining follows the same as the proof of Lemma 12.

Lemma 14. There are two positive constants $C_{1}$ and $C_{2}$, such that

$$
\sup _{\mathbf{0} \neq \mathcal{V}_{\sim} \in \boldsymbol{V}_{h D}^{\mathrm{bl}+\mathrm{bq}}} \frac{\left(\operatorname{div}{\underset{\sim}{\mathcal{v}}}_{h}, p\right)}{\left\|\underline{\sim}_{h}\right\|_{1, \Omega}} \geqslant C_{1}\|p\|_{0, \Omega}-C_{2}\|p\|_{h}, \quad p \in \mathbb{P}_{1} .
$$

Proof. Let $p \in \mathbb{P}_{1}$ be arbitrary. Then there exists $\underset{\sim}{\mathcal{w}} \in \boldsymbol{H}_{D}^{1}(\Omega)$, such that

$$
(\operatorname{div} \underset{\sim}{w}, p) \geqslant C\|p\|_{0, \Omega}^{2}
$$

and

$$
\|\underline{\sim}\|_{1, \Omega} \leqslant\|p\|_{0, \Omega}
$$

It is standard to find $\underset{\sim}{\widetilde{\mathcal{W}}} \in{\underset{\sim}{\mathcal{v}}}_{h}$ such that

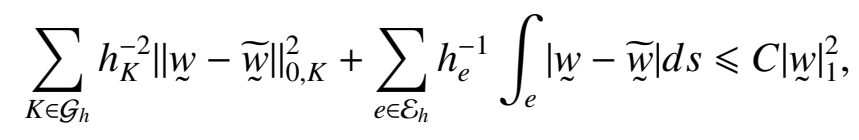


and

$$
\|\widetilde{\sim}\|_{1, \Omega} \leqslant C_{5}\|\underset{\sim}{w}\|_{1, \Omega}
$$

Integrating by parts on each $K$, and using (26) and (28), we get

$$
\begin{aligned}
& (\operatorname{div} \underset{\sim}{\widetilde{w}}, p)=(\operatorname{div}(\underset{\sim}{\widetilde{w}}-\underset{\sim}{w}), p)+(\operatorname{div} \underset{\sim}{w}, p) \geqslant(\operatorname{div}(\underset{\sim}{\widetilde{w}}-\underset{\sim}{w}), p)+C_{3}\|p\|_{0, \Omega}^{2} \\
& =\sum_{K \in \mathcal{G}_{h}}(\underset{\sim}{w}-\underset{\sim}{\widetilde{w}}, \nabla p)_{K}+\sum_{e \in \mathcal{E}_{h}} \int_{e}((\underset{\sim}{\widetilde{w}}-\underset{\sim}{w}) \cdot \mathbf{n}) \llbracket p \rrbracket d s+C_{3}\|p\|_{0, \Omega}^{2} \\
& \geqslant-\left(\sum_{K} h_{K}^{-2}\|\underset{\sim}{w}-\underset{\sim}{\widetilde{w}}\|_{0, K}+\sum_{e \in \mathcal{E}_{h}} h_{e}^{-1} \int_{e}|\boldsymbol{w}-\widetilde{\boldsymbol{w}}|^{2} d s\right)^{1 / 2}\|p\|_{h}+C_{3}\|p\|_{0, \Omega}^{2} \\
& \geqslant-C_{4}|w|_{1}\|p\|_{h}+C_{3}\|p\|_{0, \Omega}^{2} \geqslant-C_{4}\|p\|_{0, \Omega}\|p\|_{h}+C_{3}\|p\|_{0, \Omega}^{2} \\
& =\left(C_{3}\|p\|_{0, \Omega}-C_{4}\|p\|_{h}\right)\|p\|_{0, \Omega} \text {. }
\end{aligned}
$$

Equations (29) and (27) now give

$$
\|\widetilde{\widetilde{W}}\|_{1, \Omega} \leqslant C_{5}\|\underset{\sim}{\mathcal{w}}\|_{1, \Omega} \leqslant C_{5}\|p\|_{0, \Omega}
$$

Hence (30) and (31) give the asserted estimate.

Lemma 15. There is a constant $C$, such that

$$
\sup _{\underline{\sim}_{h} \in V_{h D}^{\mathrm{bl}+\mathrm{bq}}} \frac{\left(q_{h}, \operatorname{div} \underline{\sim}_{h}\right)}{\left|\underline{\sim}_{h}\right|_{1, \Omega}} \geqslant C\left\|q_{h}\right\|_{h}, \quad \forall q_{h} \in Q_{h} .
$$

Proof. By assumptions, denote $\mathcal{M}_{h}^{1}:=\left\{M \in \mathcal{M}_{h}: M\right.$ is of Type $\left.\mathrm{N}\right\}$, and $\mathcal{M}_{h}^{2}=\mathcal{M}_{h} \backslash \mathcal{M}_{h}^{1}$.

Let $q_{h} \in Q_{h}$. Given $M \in \mathcal{M}_{h}^{2}$, there exists a ${\underset{\sim}{M}}_{M} \in \boldsymbol{V}_{M 0}^{\mathrm{bl}+\text {,bq }}$, such that

$$
\left.\left(q_{h}, \operatorname{div}_{\sim}\right)_{M}\right)_{M} \geqslant C\left|q_{h}\right|_{M}^{2}
$$

and

$$
\left|{\underset{\sim}{M}}_{M}\right|_{1} \leqslant\left|q_{h}\right|_{M}
$$

Given $M \in \mathcal{M}_{h}^{1}$, there is $\underset{\sim}{{ }} \in \boldsymbol{V}_{M D}^{\mathrm{bl}+\text { bq }}$, such that

$$
\left(q_{h}, \underset{\sim}{\operatorname{div}} \underset{\sim}{v_{M}}\right)_{M} \geqslant C\left\|q_{h}\right\|_{M}^{2}
$$

and

$$
\left|v_{M}\right|_{1} \leqslant\left\|q_{h}\right\|_{M}
$$


Now define

$$
\underset{\sim}{\nu}=\sum_{M \in \mathcal{M}_{h}} \underset{\sim}{v_{M}}
$$

Then

$$
\left(\operatorname{div} \underset{\sim}{v}, q_{h}\right)=\sum_{M \in \mathcal{M}_{h}}\left(\underset{\sim}{\operatorname{div}} \underset{\sim}{M}, q_{h}\right)_{M} \geqslant C\left(\sum_{M \in \mathcal{M}_{h}^{1}}\left\|q_{h}\right\|_{M}^{2}+\sum_{M \in \mathcal{M}_{h}^{2}}\left|q_{h}\right|_{M}^{2}\right)
$$

and

$$
|\underline{\sim}|_{1}^{2}=\sum_{M \in \mathcal{M}_{h}}\left|\underline{\sim}_{M}\right|_{1}^{2} \leqslant \sum_{M \in \mathcal{M}_{h}^{1}}\left\|q_{h}\right\|_{M}^{2}+\sum_{M \in \mathcal{M}_{h}^{2}}\left|q_{h}\right|_{M}^{2}
$$

Therefore,

$$
\frac{\left(\operatorname{div} \underset{\sim}{\mathcal{v}}, q_{h}\right)}{|\underline{\sim}|_{1}} \geqslant C\left(\sum_{M \in \mathcal{M}_{h}^{1}}\left\|q_{h}\right\|_{M}^{2}+\sum_{M \in \mathcal{M}_{h}^{2}}\left|q_{h}\right|_{M}^{2}\right)^{1 / 2}
$$

Since every $e \in \mathcal{E}_{h}^{N}$ is covered by at least one $M \in \mathcal{M}_{h}^{1}$, we have

$$
\sum_{M \in \mathcal{M}_{h}^{1}}\left\|q_{h}\right\|_{M}^{2}+\sum_{M \in \mathcal{M}_{h}^{2}}\left|q_{h}\right|_{M}^{2} \geqslant \sum_{M \in \mathcal{M}_{h}}\left|q_{h}\right|_{M}^{2}+\sum_{e \in \mathcal{E}_{h}^{D}} h_{e} \int_{e} \llbracket q_{h}\left\|^{2} \gtrsim\right\| q_{h} \|_{h}^{2}
$$

This finishes the proof.

Proof of Theorem 11. Let $C_{1}$ and $C_{2}$, and $C_{3}$ be given in Lemmas 14 and 15, respectively. Given $\xi \in(0,1)$, then

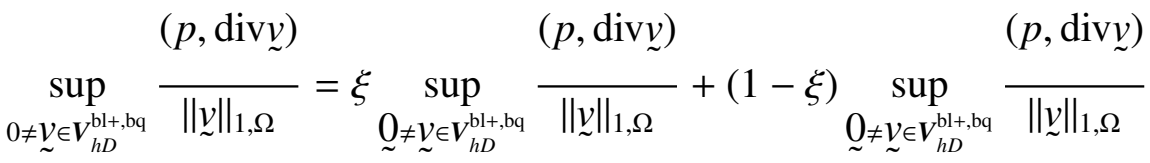

$$
\begin{aligned}
& \geqslant C_{1} \xi\|p\|_{0, \Omega}+\left[(1-\xi) C_{3}-\xi C_{2}\right]\|p\|_{h} .
\end{aligned}
$$

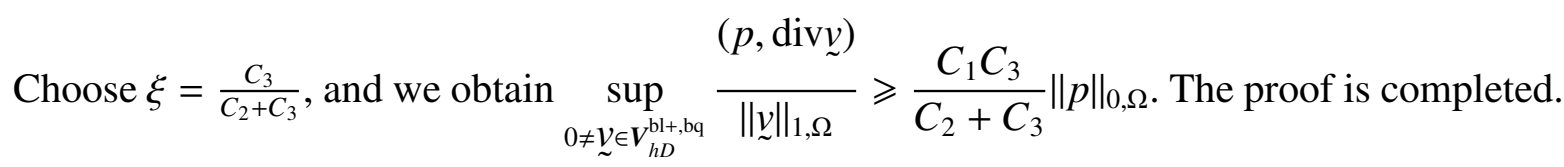

Remark 16. Symmetrically, we can prove the inf-sup stability of the finite element pair $\boldsymbol{V}_{h N}^{\mathrm{bl+}, \mathrm{bq}}-\mathbb{P}_{1}$. Also, we can define symmetrically the finite element spaces $\boldsymbol{V}_{h D}^{\mathrm{bq}, \mathrm{bl}+}$ and $\boldsymbol{V}_{h N}^{\mathrm{bq}, \mathrm{bl}+}$, and prove the infsup stability of the pairs $\boldsymbol{V}_{h D}^{\mathrm{bq}, \mathrm{bl}+}-\mathbb{P}_{1}$ and $\boldsymbol{V}_{h N}^{\mathrm{bq}, \mathrm{bl+}}-\mathbb{P}_{1}$.

Remark 17. Noting that the degree of $\operatorname{div} \mathbf{V}_{h}^{\mathrm{bl+}+\mathrm{bq}}$ is higher than $\mathbb{P}_{1}$, we can not expect the conservation property SC 3 holds. 
4. Stability CONDITIONS OF THE FINITE ELEMENT PAIR $\boldsymbol{V}_{h D}^{\mathrm{LTZ}, \mathrm{bl} \pm}-\mathbb{P}_{1}$

In this section, we prove the stability conditions SC 1, SC 2 and SC 3 for the $\boldsymbol{V}_{h D}^{\mathrm{LTZ}, \mathrm{bl} \pm}-\mathbb{P}_{1}$ pair. The condition SC 1 is constructed in the theorem below.

Theorem 18. There exists a constant $C$ depending on $\Omega, \Gamma_{D}, \Gamma_{N}$ and the shape regularity of $\mathcal{G}_{h}$ only, such that

$$
\inf _{q_{h} \in \mathbb{P}_{1}} \sup _{\mathcal{v}_{h} \in V_{h D}^{\mathrm{LTZ}, \mathrm{bl} \pm}} \frac{\left(q_{h}, \operatorname{div}_{\sim}{\underset{\sim}{h}}_{h}\right)}{\left\|q_{h}\right\|_{0, \Omega}\left\|v_{\sim}\right\|_{1, \Omega}} \geqslant C .
$$

Theorem 18 can be proved by repeating the proof of Theorem 11. We only have to note that, in contrast to the $\boldsymbol{V}_{h D}^{\mathrm{bl}+\text {,bq }}$ element, the $\boldsymbol{V}_{h D}^{\mathrm{LTZ}, \mathrm{bl} \pm}$ element falls into the category of nonconforming ones, which may bring in some technical difficulty in repeating (30), which can be circumvented by standard techniques. As evidently $\operatorname{div}_{h} \boldsymbol{V}_{h D}^{\mathrm{bl}+\text {,bq }} \subset \mathbb{P}_{1}$, the condition $\mathbf{S C} 3$ follows immediately.

Now it remains to us to prove the condition SC 2, namely the discrete Korn's inequality. We have the theorem below.

Theorem 19. The Korn's inequality holds that

$$
\left\|\underline{\sim}_{h}\right\|_{1, h} \leqslant C\left\|\boldsymbol{\varepsilon}_{h}\left({\underset{\sim}{h}}_{h}\right)\right\|_{0, \Omega}, \quad \forall{\underset{\sim}{v}}_{h} \in \boldsymbol{V}_{h D}^{\mathrm{LTZ}, \mathrm{bl} \pm}
$$

Proof. Given ${\underset{\sim}{h}}_{h} \in \boldsymbol{V}_{h D}^{\mathrm{LTZ} \text {,bl } \pm}$ arbitrarily, it holds on any $K \in \mathcal{G}_{h}$ that

$$
\boldsymbol{\varepsilon}\left({\underset{\sim}{h}}_{h}\right)=\left(\nabla{\underset{\sim}{v}}_{h}-\frac{1}{2} \operatorname{rot}_{\sim} \underset{\sim}{ } \chi\right), \text { with } \chi=\left[\begin{array}{cc}
0 & -1 \\
1 & 0
\end{array}\right]
$$

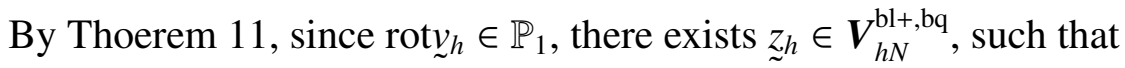

$$
\sum_{K \in \mathcal{G}_{h}} \int_{K} \operatorname{div}{\underset{\sim}{z_{h}}}_{h} \operatorname{rot}{\underset{\sim}{h}}_{h}=\sum_{K \in \mathcal{G}_{h}} \int_{K} \operatorname{rot}{\underset{\sim}{h}}_{h} \operatorname{rot}{\underset{\sim}{h}}_{h},
$$

and

$$
\|z\|_{1, \Omega} \lesssim\left\|\operatorname{rot}_{h}{\underset{\sim}{h}}_{h}\right\|_{0, \Omega} \leqslant\left|{\underset{\sim}{v}}_{h}\right|_{1, h}
$$


Denote ${\underset{\sim}{h}}_{h}:=\nabla_{h} \underset{\sim}{\mathcal{v}_{h}}-\operatorname{curl} \underset{\sim}{{ }_{h}}$. Then

$$
\begin{aligned}
& \sum_{K \in \mathcal{G}_{h}} \int_{K} \boldsymbol{\varepsilon}\left({\underset{\sim}{v} h}_{h}\right):{\underset{\sim}{\tau} h}_{h} d x=\sum_{K \in \mathcal{G}_{h}} \int_{K}\left(\nabla{\underset{\sim}{v}}_{h}-\frac{1}{2} \operatorname{rot}{\underset{\sim}{h}}_{h} \chi\right):\left(\underset{\sim}{v_{h}}-\operatorname{curl}{\underset{\sim}{\sim} h}_{h}\right) d x \\
& (\operatorname{noting}(36))=\sum_{K \in \mathcal{G}_{h}} \int_{K}\left|\nabla \underset{\sim}{v_{h}}\right|^{2} d x-\int_{K \in \mathcal{G}_{h}} \nabla_{\sim} \underset{h}{ } \operatorname{curl} z_{\sim} \text {. }
\end{aligned}
$$

Integrating by parts one obtains

$$
\sum_{K \in \mathcal{G}_{h}} \int_{K} \nabla \underset{\sim}{v_{h}}: \operatorname{curl}{\underset{\sim}{h} h}_{T \in \mathcal{G}_{h}} d x=\int_{\partial K}\left(v_{1} \nabla z_{1} \cdot \mathbf{t}_{K}+v_{2} \nabla z_{2} \cdot \mathbf{t}_{K}\right) d s,
$$

where $\mathbf{t}_{K}$ is the counterclockwise tangent to $\partial K$. Now, let us inspect the different contributions to the the sum of integral, on the righthand side above. First, we recall that $\underset{\sim}{z_{h}}=\underset{\sim}{0}$ on $\Gamma_{N}$. Hence, the contribution from the edges lying on the boundary part $\Gamma_{N}$ vanish. Secondly, consider the contribution from interior edges. Let $e=K_{L} \cap K_{R}$ be the common edge of two adjoining cells $K_{L}$ and $K_{R}$ in $\mathcal{G}_{h}$. On the edge it holds, by the continuity of $z_{i}$,

$$
\nabla z_{i} \cdot \mathbf{t}_{K_{L}}+\nabla z_{i} \cdot \mathbf{t}_{K_{R}}=0, \quad i=1,2
$$

Since the component $v_{2}$ is first-order moment continuous, and $\nabla z_{2} \cdot \tau_{e} \in P_{1}(e)$, this directly gives

$$
\left.\int_{e} v_{2}\right|_{K_{L}} \nabla z_{2} \cdot \tau_{K_{L}} d s+\left.\int_{e} v_{2}\right|_{K_{R}} \nabla z_{2} \cdot \tau_{K_{R}} d s=\int_{e} \llbracket v_{2} \rrbracket \nabla z_{2} \cdot \tau_{e} d s=0
$$

Since $\nabla z_{1} \cdot \mathbf{t}_{K_{i}}, i=1,2$, are constants with opposite values, and $v_{1}$ is zero-order moment continuous, we get

$$
\left.\int_{e} v_{1}\right|_{K_{L}} \nabla z_{1} \cdot \tau_{K_{L}} d s+\left.\int_{e} v_{1}\right|_{K_{R}} \nabla z_{2} \cdot \tau_{K_{R}} d s=\int_{e} \llbracket v_{1} \rrbracket \nabla z_{1} \cdot \tau_{e} d s=0
$$

Therefore, the contribution from interior edges in the sum of integrals in the right hand side of (38) vanish. Thirdly and finally, on an edge $e \subset \Gamma_{D}$ it holds that the first order moment of $v_{2}$ and the zero-order moment of $v_{1}$ vanish, since $\nabla z_{2} \cdot \tau_{e} \in P_{1}(e)$, and $\nabla z_{1} \cdot \tau_{e} \in P_{0}(e)$, the contributions to the right hand side of (38) by edges on $\Gamma_{D}$ also vanish. We have now proved that

$$
\sum_{K \in \mathcal{G}_{h}} \int_{K} \nabla{\underset{\sim}{h}}_{h}: \operatorname{curl}{\underset{\sim}{h} h}_{h} d x=0
$$

And we thus obtain

$$
\sum_{K \in \mathcal{G}_{h}} \int_{K} \boldsymbol{\varepsilon}\left(\underline{\sim}_{h}\right): \tau d x=\left|\underline{\sim}_{\sim}\right|_{1, h}^{2}
$$


Noting that

$$
\left\|\tau_{\sim}\right\|_{0, \Omega} \leqslant\left|{\underset{\sim}{h}}_{h}\right|_{1, h}+\left|\underset{\sim}{z_{h}}\right|_{1, \Omega} \leqslant\left.\left. C\right|_{\sim}\right|_{1, h}
$$

we obtain

$$
\left(\sum_{K \in \mathcal{G}_{h}} \int_{K}\left|\boldsymbol{\varepsilon}\left(\underline{\sim}_{h}\right)\right|^{2} d x\right)^{1 / 2} \geqslant \sum_{K \in \mathcal{G}_{h}} \int_{K} \boldsymbol{\varepsilon}_{h}\left({\underset{\sim}{\nu}}_{h}\right):{\underset{\sim}{\tau} h}_{h} d x /\left\|\tilde{\sim}_{h}\right\|_{0, \Omega} \geqslant C\left|{\underset{\sim}{\nu}}_{h}\right|_{1, h} .
$$

The proof is completed.

Remark 20. We only prove (34) for ${\underset{\sim}{h}}_{h} \in \boldsymbol{V}_{h D}^{\mathrm{LTZ}, \mathrm{bl} \pm}$, and the proof of that for ${\underset{\sim}{h}}_{h} \in \boldsymbol{V}_{h N}^{\mathrm{LTZ}, \mathrm{bl} \pm}$ is the same. Also symmetrically, we can prove the stability conditions SC 1, SC 2 and $\mathbf{S C} \mathbf{3}$ for the pairs $\boldsymbol{V}_{h N}^{\mathrm{LTZ}, \mathrm{bl} \pm}-\mathbb{P}_{1}, \boldsymbol{V}_{h D}^{\mathrm{bl} \pm \mathrm{LTZ}}-\mathbb{P}_{1}$ and $\boldsymbol{V}_{h N}^{\mathrm{bl} \pm \mathrm{LTZ}}-\mathbb{P}_{1}$.

\section{Numerical EXAMPLES}

In this section, we present some numerical examples, which verify the theoretical analysis above. In this examples, the computational domains are all $\Omega=(0,1) \times(0,1)$, and we set $\Gamma_{N}=[0,1] \times\{0\}$ and $\Gamma_{D}=\overline{\partial \Omega \backslash \Gamma_{N}}$. We divide the domain into $M \times M$ squares and obviously $h=\sqrt{2} / M$. We test both the cases that the essential boundary condition is imposed on $\partial \Omega$, which is the pure Dirichlet boundary condition, and that the essential boundary condition is imposed on $\Gamma_{D}$ only, which is the mixed boundary condition case, respectively. In every figure below, the left part shows the results with respect to the pure Dirichlet boundary condition and the right for the mixed boundary condition.

5.1. The numerical examples for linear elasticity. We firstly test the locking-free performance of the element for linear elasticity. In the series of numerical experiments, we choose $\underset{\sim}{f}$ such that

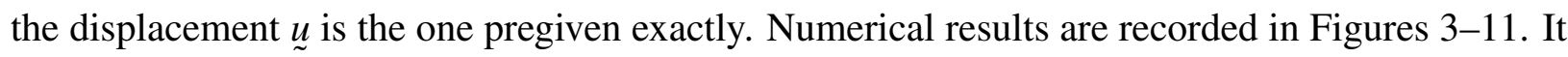
illustrates the robustness of the finite element with respect to $\lambda$. In Figures 3-11 the x-label shows the value of $\log _{2} M$ and the $y$-label shows the value of the log base 2 of error.

(1) In Figures 3, 4, and 5, the displacement is $\underset{\sim}{1}=\left(1+2 x+4 y+3 x^{2}+7 x y+8 y^{2}, 1+3 x+\right.$ $\left.5 y+6 x^{2}+9 x y+10 y^{2}\right)^{\top}$, and $\lambda$ varies;

(2) in Figures 6, 7, and 8, the displacement is $\underset{\sim}{u^{2}}=\left(x^{3}+8 x^{2} y+7 x y^{2}+10 y^{3}, 10 x^{3}+3 x^{2} y+\right.$ $\left.x y^{2}+2 y^{3}\right)^{\top}$, and $\lambda$ varies;

(3) in Figures 9, 10, and 11, the displacement is $\underset{\sim}{\underline{u}}{ }^{3}=(\sin (x+y), \sin (x y))^{\top}$, and $\lambda$ varies. 
(4) in Figures 12 ,the displacement is $\underset{\sim}{u}=\left(100(x(x-1))^{3}(y(y-1))^{2}(2 y-1),-100(y(y-\right.$ $\left.1))^{3}(x(x-1))^{2}(2 x-1)\right)^{\top}$. The experiment is carried on a fixed grid with $M=128$, and $\lambda$ varies. In the figure, the $x$-label shows the value of $\log _{2} \lambda$
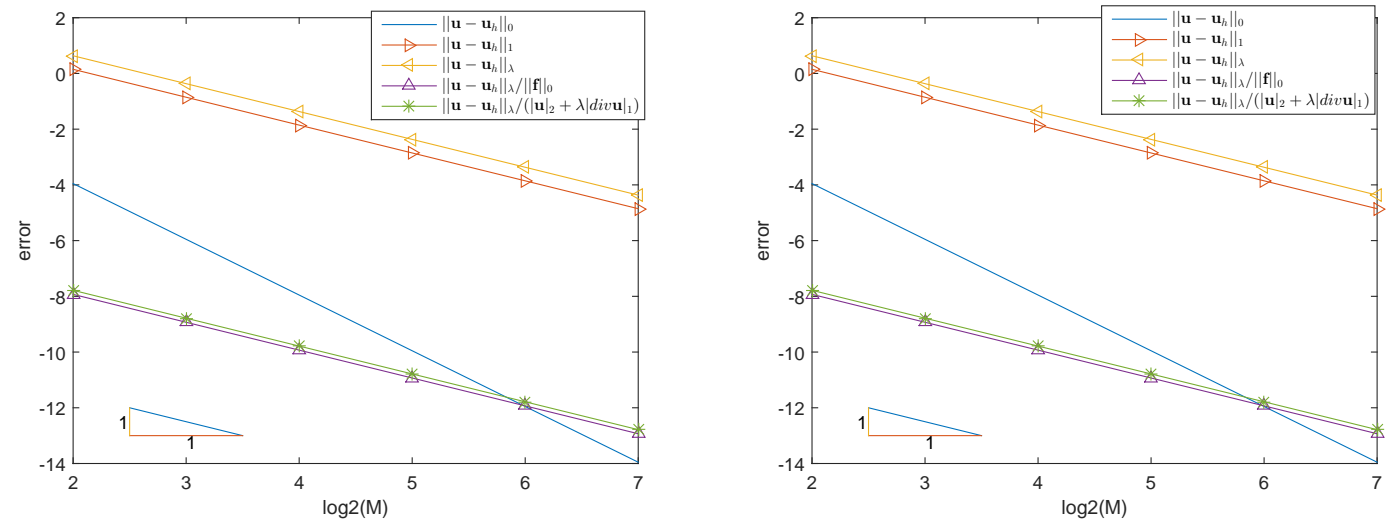

FIGURE 3. Linear elasticity, example (1), and $\lambda=10$
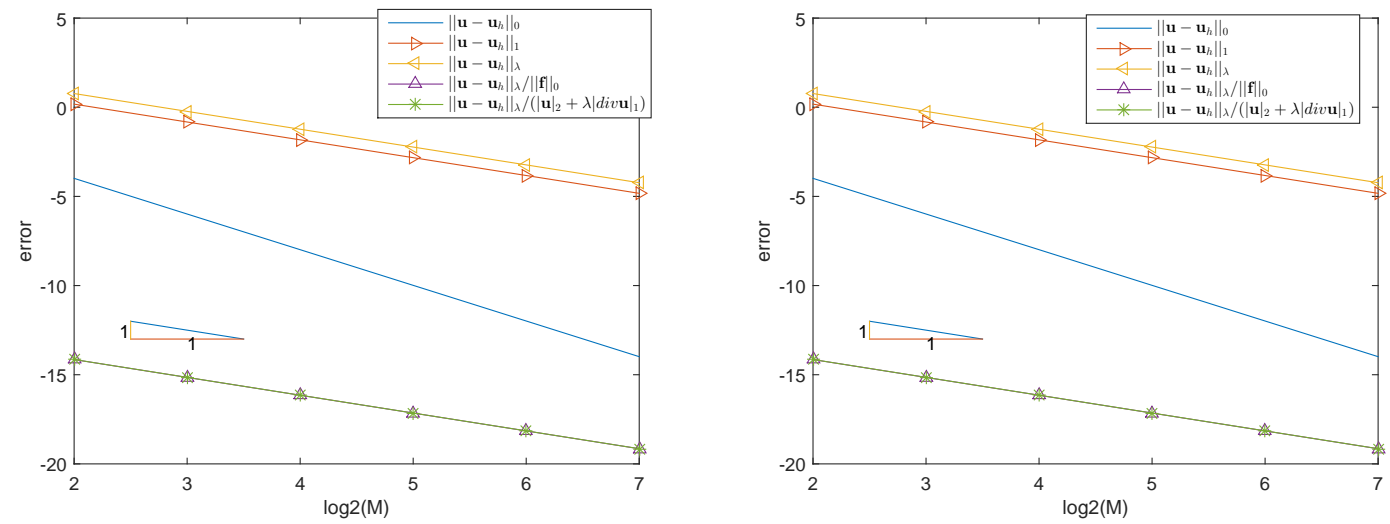

FIgURE 4. Linear elasticity, example (1), and $\lambda=10^{3}$

5.2. The numerical examples for Stokes problem. We then test the performance of the element pair for incompressible Stokes problem. In the series of numerical experiments, again, we choose $\underset{\sim}{f}$ such that the velocity $\underset{\sim}{u}$ and pressure $p$ are the ones pregiven exactly. Numerical results are recorded in Figures 13 and 14. It illustrates the performance of the element pair.

(1) In Figure 13, the velocity is $\underset{\sim}{\sim}{ }^{1}=\left(x^{3}+8 x^{2} y-6 x y^{2}+10 y^{3}, 10 x^{3}-3 x^{2} y-8 x y^{2}+2 y^{3}\right)^{\top}$, and the pressure is $p=4 x^{3}-6 x y^{2}$. 

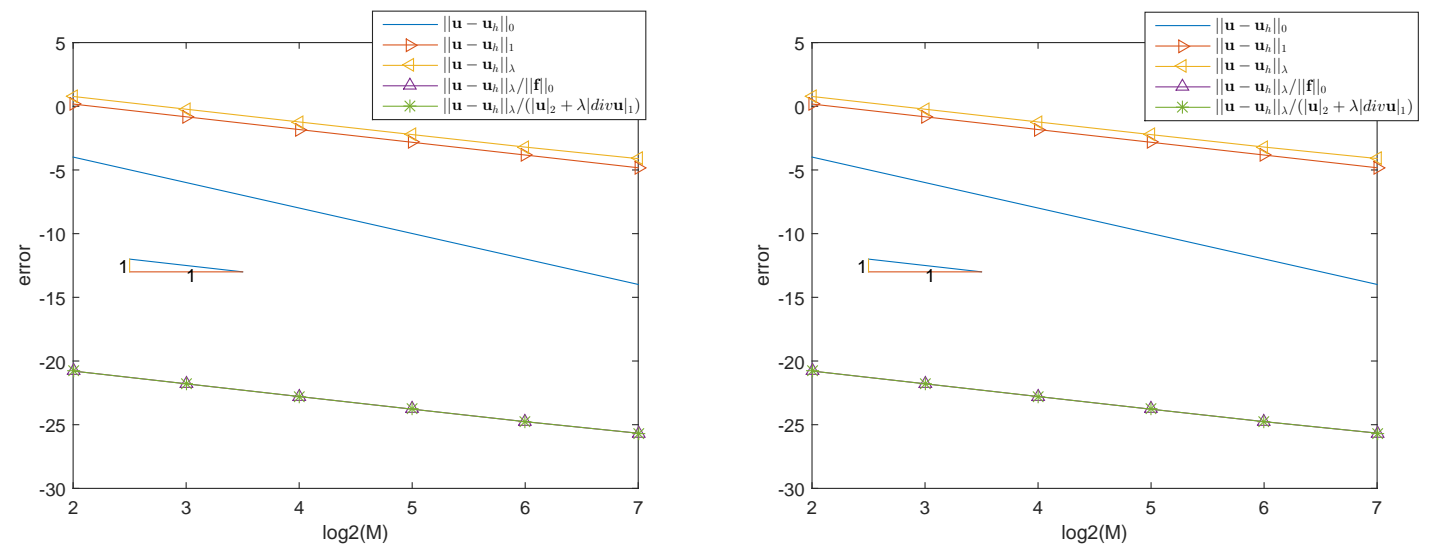

FIGURE 5. Linear elasticity, example (1), and $\lambda=10^{5}$
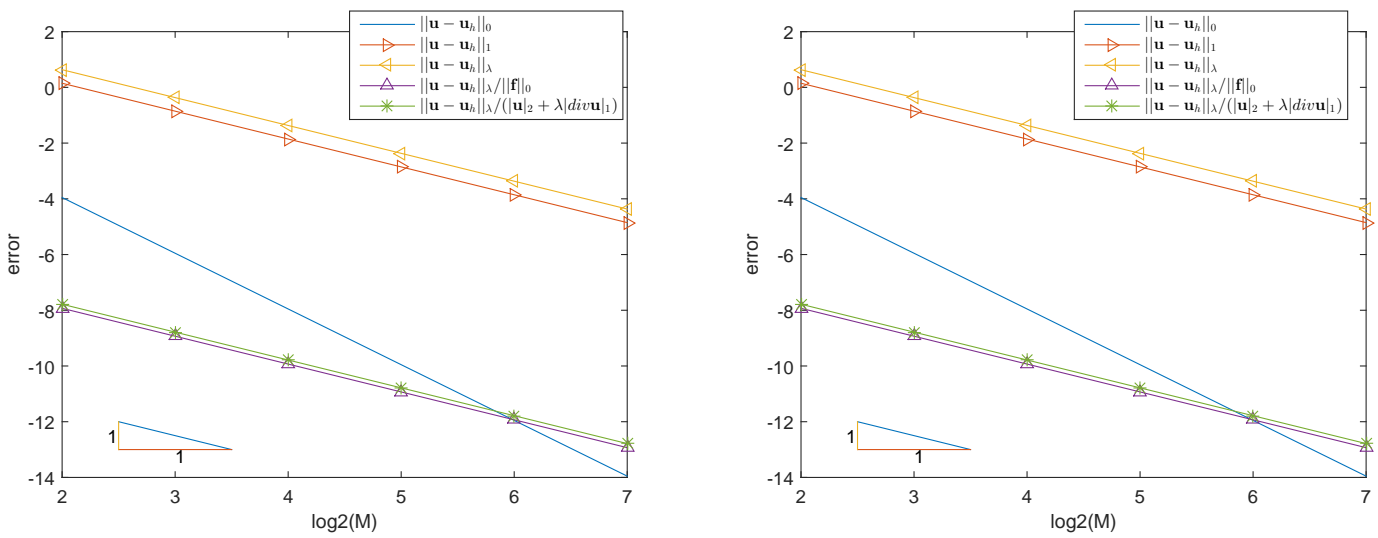

FiguRe 6. Linear elasticity, example (2), and $\lambda=10$
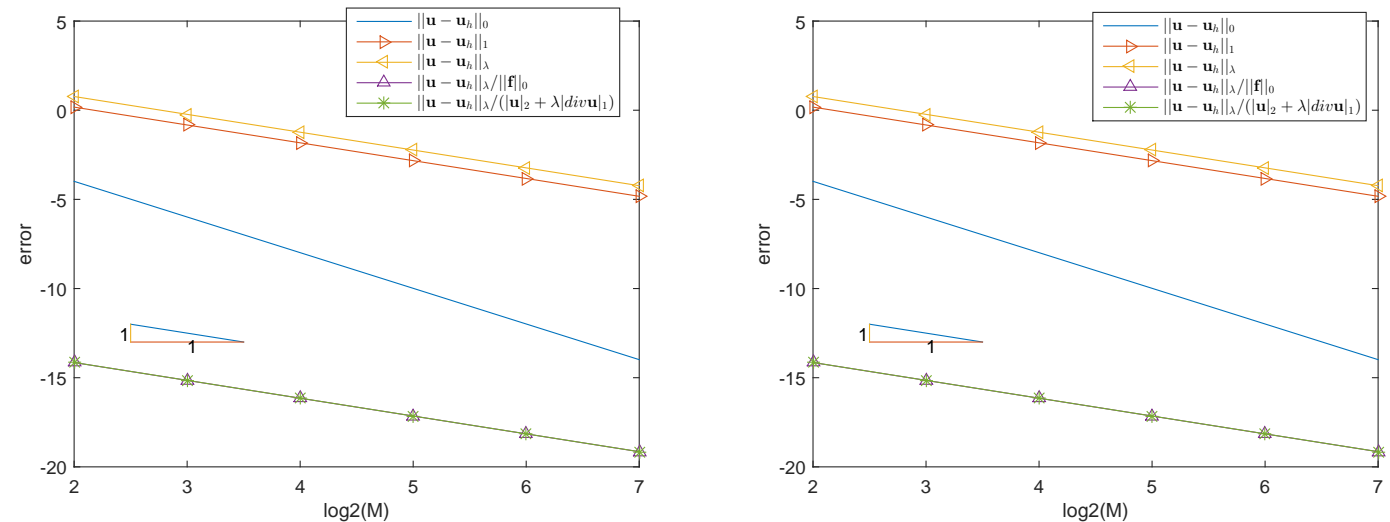

Figure 7. Linear elasticity, example (2), and $\lambda=10^{3}$

(2) In Figure 14, the velocity is ${\underset{\sim}{u}}^{2}=(\cos x \cos y, \sin x \sin y)^{\top}$, and the pressure is $p=4 x^{3}-$ $6 x y^{2}$. 

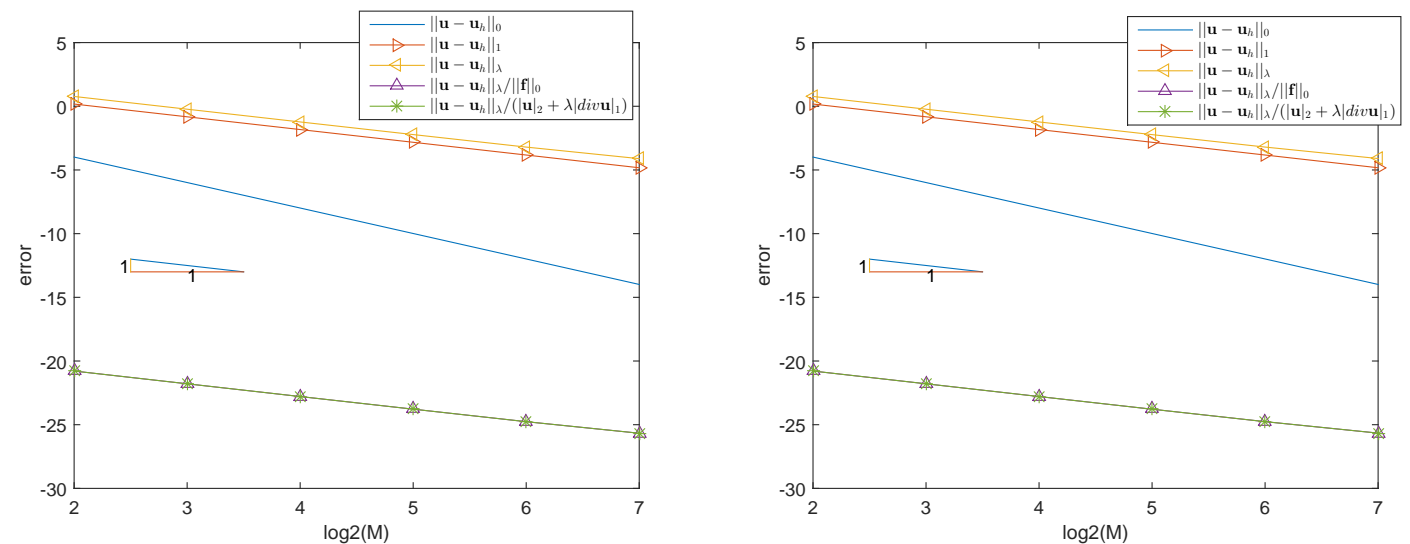

FIGURE 8. Linear elasticity, example (2), and $\lambda=10^{5}$
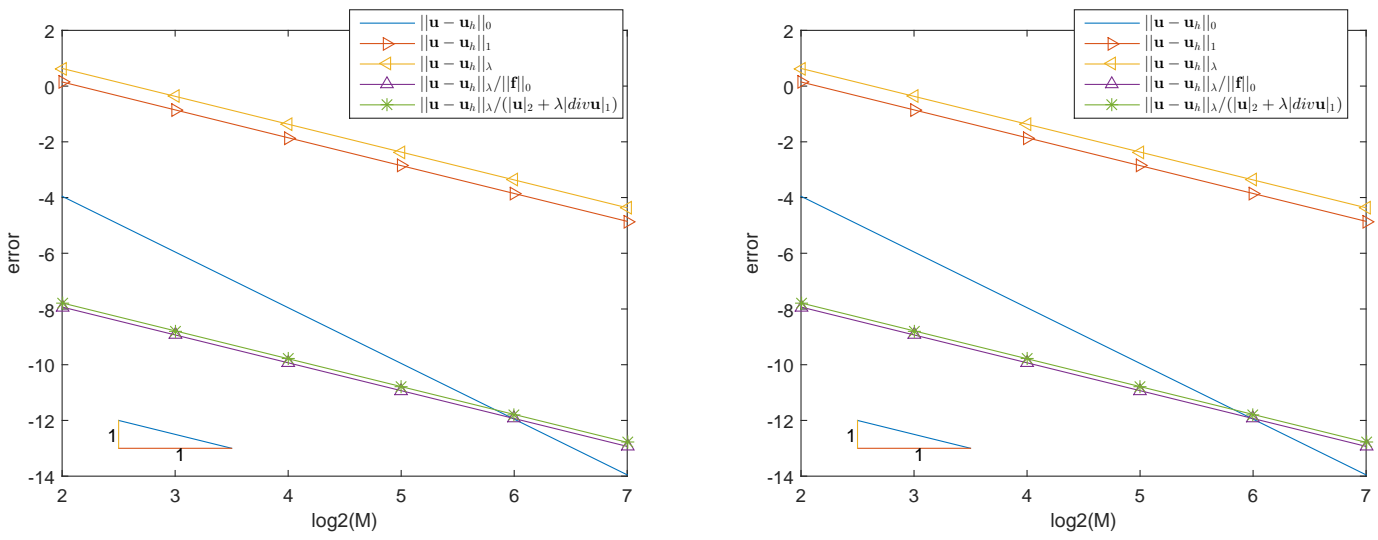

FIGURE 9. Linear elasticity, example (3), and $\lambda=10$
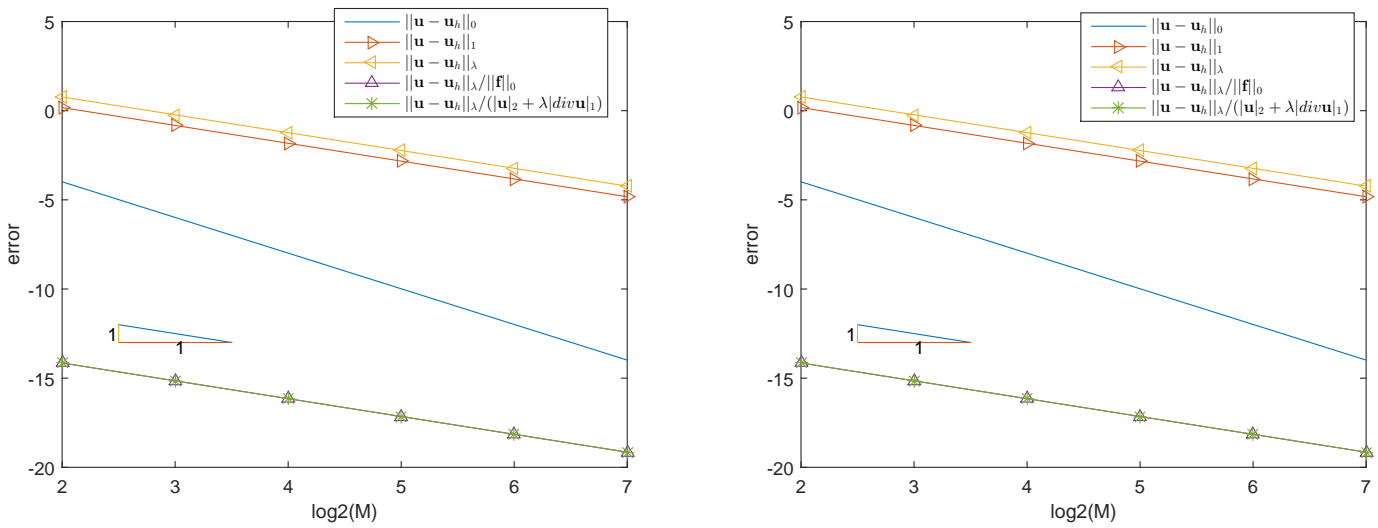

FIgURE 10. Linear elasticity, example (3), and $\lambda=10^{3}$

\section{Concluding Remarks}

In this paper, we construct a stable pair for the incompressible Stokes flow and nearly linear elasticity on rectangular grids. The stable conditions SC 1, SC 2 and SC 3 are verified. As far as 

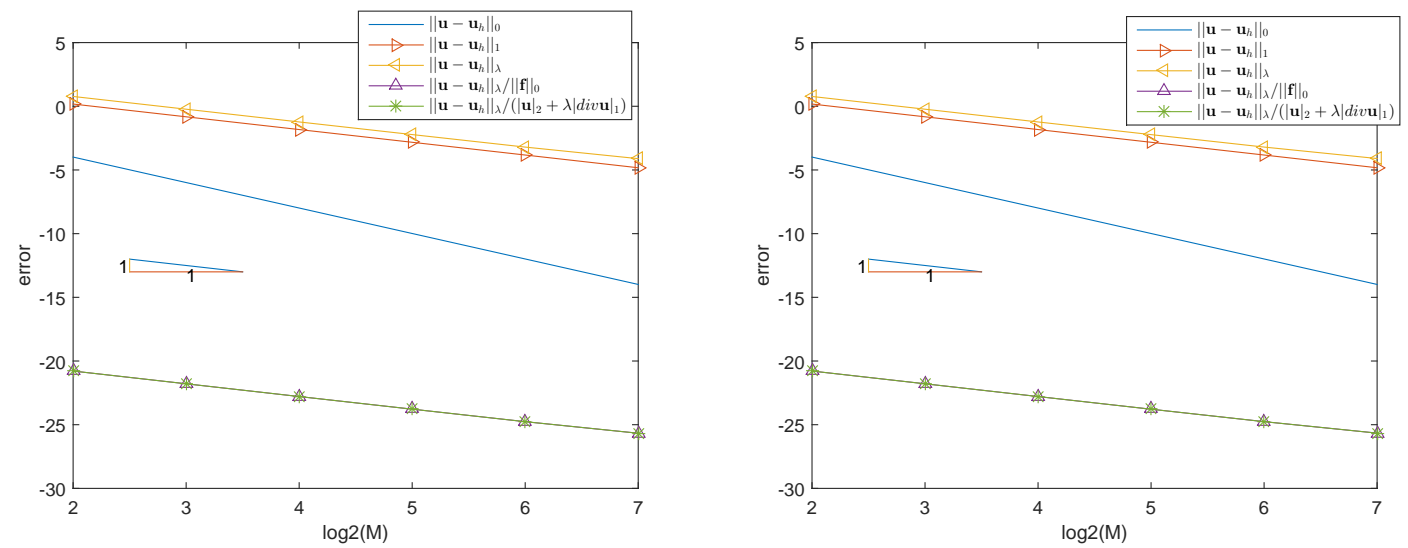

FIgURE 11. Linear elasticity, example (3), and $\lambda=10^{5}$
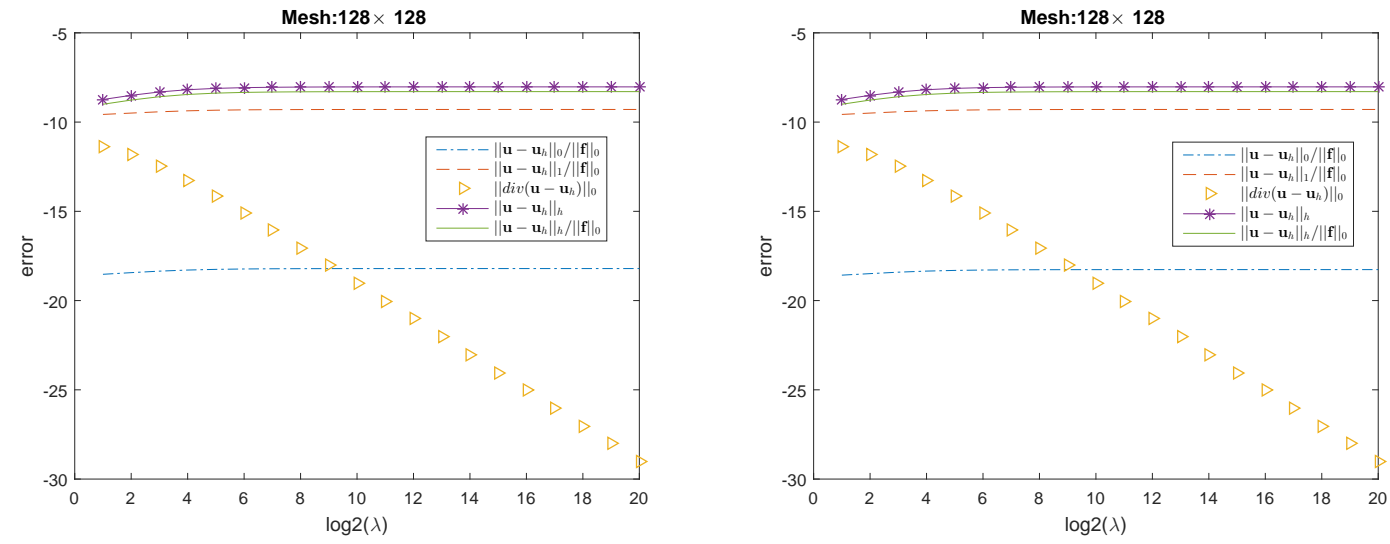

FIGURE 12. Linear elasticity, example (4), and $M=128$
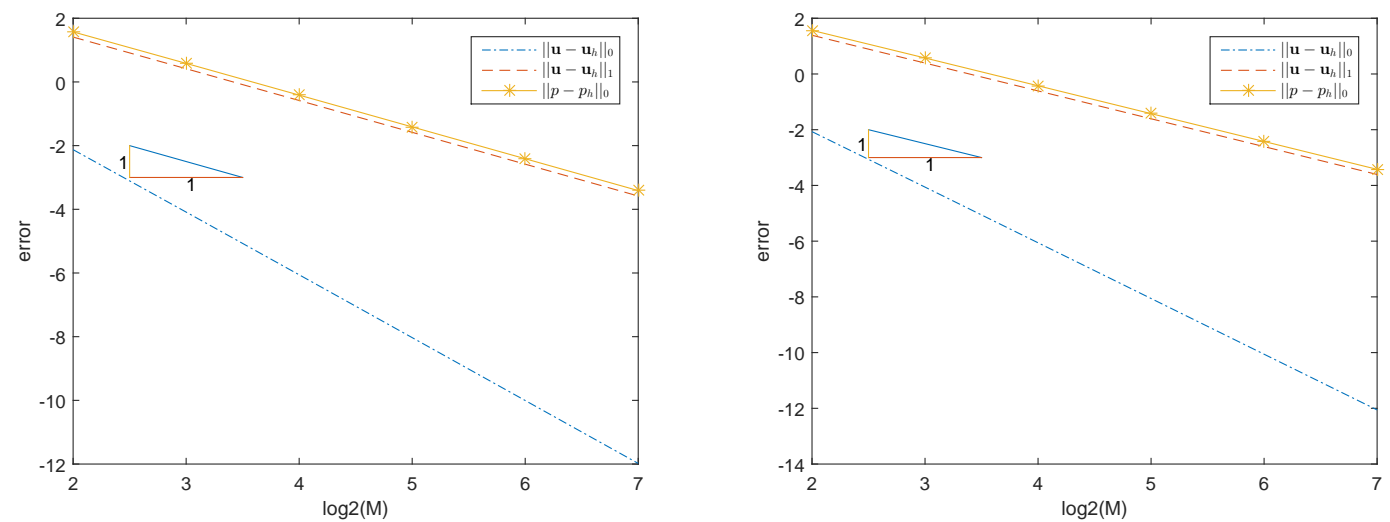

Figure 13. Stokes example (1).

we know, it is of the lowest degree among the finite element pairs on rectangular grids for which the stable conditions SC 1, SC 2 and SC 3 all hold. 

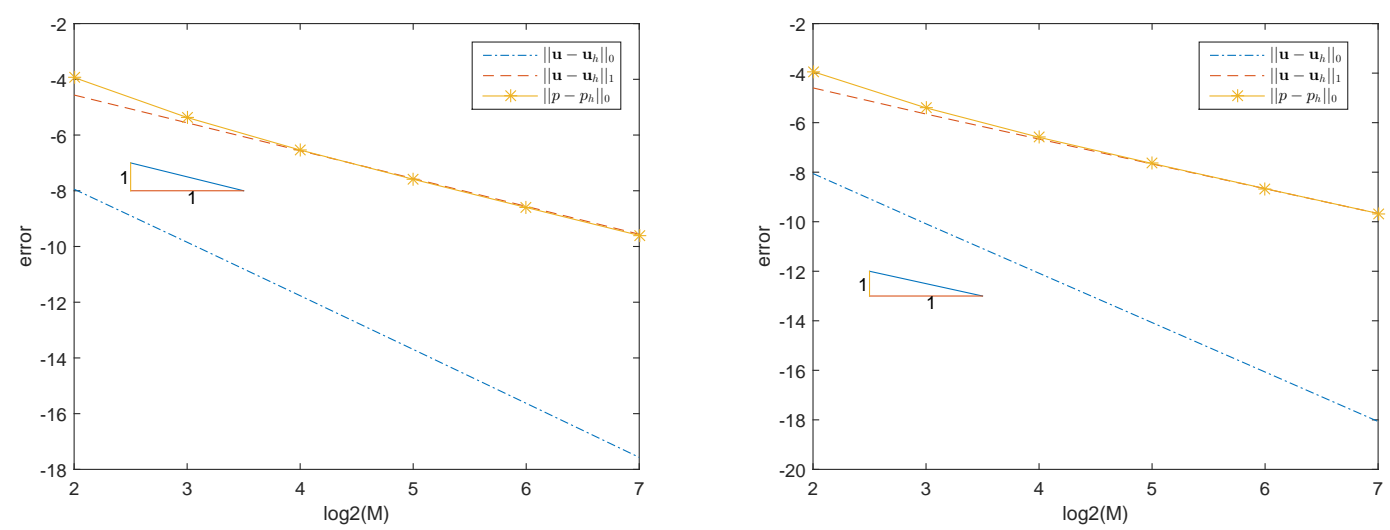

FIGURE 14. Stokes example (2).

As the two components for the velocity (or displacement) space are Lin-Tobiska-Zhou element and the nonconforming enhanced bilinear element, the super-consistency can be proved. Some high accuracy can be expected by postprocessing. This will be discussed in future works.

It is natural to discuss the connection between the present scheme and other discretizations. It has been realized more and more clear that discrete complexes of various kinds can play a key role in designing numerical algorithms. The utilisation of discrete complexes can be found in, e.g., designing structure-preserving discretisation and fast solvers(c.f. [44]). There have been works that introduced the methodology onto linear elasticity problem (c.f., $[1,3]$ and references therein). As to the pair presented in this paper, that both SC 1 and SC 3 hold hints us there can be a discrete Stokes complex associated. The existence and application of such a discrete Stokes complex will be studied in future. There are discretisations of the linear elasticity problem in other formulation and by other variational principles(c.f., e.g., $[39,46,54]$ ), and also related linear elasticity problems like the Reissner-Mindlin plate problem. The comparison will also be discussed in future.

Acknowledgement. The authors would like to thank the anonymous referee for the valuable comments which help improve the manuscript. The author Y. C. is partially supported by National Science Foundation of China(NSFC) under grant NSFC 91430215; the author S. Z. is partially supported by NSFC 11101415 and NSFC 11471026, and the SRF for ROCS, SEM, China.

\section{REFERENCES}

[1] Angoshtari A., Yavari A.: A geometric structure-preserving discretization scheme for incompressible linearized elasticity, Comput. Methods Appl. Mech. Engrg. 259(2013), 130-153.

[2] Araya R., Barrenechea G., Valentin F.: Stabilized finite element methods based on multiscale enrichment for the stokes problem, SIAM J. NUMER. ANAL. 44(2006), 322-348.

[3] Arnold D.N., Winther R.: Mixed finite elements for elasticity, Numer. Math. 92(2002) 401-419. 
[4] Arnold D.N., Qin J.: Quadratic velocity/linear pressure Stokes elements, in Proceedings of Advances in Computer Methods for Partial Differential Equations VII, R. Vichnevetsky and R. S. Steplemen, eds., AICA, 1992.

[5] Auricchio F., Beirão da Veiga L., Lovadina C., Reali A.: The importance of the exact satisfaction of the incompressibility constraint in nonlinear elasticity: mixed FEMs versus NURBS-based approximations, Comput. Methods Appl. Mech. Engrg. 199(2010), 314-323.

[6] Auricchio F., da Veiga Beirao L., Buffam A., Lovadina C., Reali A.: A fully locking-free isogeometric approach for plane linear elasticity problems: a stream function formulation, Comput. Methods Appl. Mech. Engrg. 197(2007) 160-172.

[7] Babuška I.: The finite element method with Lagrangian multipliers, Numer. Math. 20(1973), 179-192.

[8] Barrenechea G. R., Valentin F.: Consistent local projection stabilized finite element methods, SIAM J. Numer. Anal, 2010, 48(5): 1801-1825.

[9] Bejanov B., Guermond J.L., Minev P.D.: A locally DIV-free projection scheme for incompressible flows based on non-conforming finite elements, Int. J. Numer. Math. Fluids 49(2005) 549-568.

[10] Belytschko T., Lu Y.Y., Gu L.: Element-free Galerkin methods, Int. J. Numer. Methods Engrg. 37(1994), 229256.

[11] Bochev P., Lai J., Olson L.: A non-conforming least-squares finite element method for incompressible fluid flow problems, Int. J. Numer. Meth. Fluids 72(2013), 375-402.

[12] Boffi D., Brezzi F., Fortin M.: Mixed finite element methods and applications. Berlin: Springer, 2013.

[13] Boffi D., Cavallini N., Gardini F., Gastaldi L.: Local mass conservation of Stokes finite elements, J. Sci. Comput. 52(2012), 383-400.

[14] Bolton P., Thatcher R.W.: On mass conservation in least-squares methods, J. Comput. Phys. 203(2005), 287304.

[15] Braack M., Burman E.: Local projection stabilization for the Oseen problem and its interpretation as a variational multiscale method, SIAM J. Numer. Anal. 43(2006), 2544-2566.

[16] Braack M., Burman E., John V., Lube G.: Stabilized finite element methods for the generalized Oseen problem, Comput. Methods Appl. Mech. Engrg. 196(2007), 853-866.

[17] Bramble J.H., Lazarov R.D., Pasciak J.E.: Least-squares methods for linear elasticity based on a discrete minus one inner product, Comput. Methods Appl. Mech. Engrg. 191 (2001), 727-744.

[18] Brenner S., Scott R.: The mathematical theory of finite element methods, 3rd Ed, TAM Vol. 15. Springer Science \& Business Media, 2008.

[19] Brenner S.C., Sung L.Y.: Linear finite element methods for planar linear elasticity, Math. Comput. 59(1992) 321-338.

[20] Brenner S.: Korn's inequalities for piecewise H1 vector fields, Math. Comput. 73(2004), 1067-1087.

[21] Brezzi F.: On the existence, uniqueness and approximation of saddle-point problems arising from Lagrange multipliers, R.A.I.R.O. Anal. Numer. R2,(1974) 129-151.

[22] Brezzi F., Fortin M.: Mixed and Hybrid Finite Element Methods, Springer Ser. Comput. Math., 15, SpringerVerlag, New York, 1991.

[23] Burman E., Linke A.: Stabilized finite element schemes for incompressible flow using Scott-Vogelius elements, Appl. Numer. Math. 58(2008), 1704-1719. 
[24] Carrero J., Cockburn B., Schötzau D.: Hybridized globally divergence-free LDG methods. I. The Stokes problem, Math. Comp. 75(2006), 533-563.

[25] Case M.A., Ervin V.J., Linke A., Rebholz L.G.: A connection between Scott-Vogelius and grad-div stabilized Taylor-Hood FE approximations of the Navier-Stokes equations, SIAM J. Numer. Anal. 49(2011), 1461-1481.

[26] Chang C., Nelson J.: Least-Squares finite element method for the Stokes problem with zero residual of mass conservation, SIAM J. Numer. Anal. 34(1997), 480-489.

[27] Chen S., Ren G., Mao S.: Second-order locking-free nonconforming elements for planar linear elasticity, J. Comput. Appl. Math. 233 (2010), 2534-2548.

[28] Cockburn B., Kanschat G., Schötzau D.: A note on discontinuous Galerkin divergence free solutions of the Navier-Stokes equations, J. Sci. Comput. 31(2007), 61-73.

[29] Cockburn B., Kanschat G., Schötzau D.: A locally conservative LDG method for the incompressible NavierStokes equations, Math. Comp. 74(2004), 1067-1095.

[30] Cockburn B., Kanschat G., Schötzau D.: The local discontinuous Galerkin method for linearized incompressible fluid flow: a review, Comput. Fluids 34(2005), 491-506.

[31] Cousins B.R., Brone S. L., Linke A., Rebholz L.G., Wang Z.: Efficient Linear Solvers for Incompressible Flow Simulations using Scott-Vogelius Finite Elements, Numer Methods Partial Differential Eq 29(2013), 12171237.

[32] Crouzeix M., Raviart P.: Conforming and non conforming finite element methods for solving the stationary Stokes equations R.A.I.R.O. R3,(1973) 33-76.

[33] Evans J.: Divergence-free B-spline Discretizations for Viscous Incompressible Flows, PhD thesis, The University of Texas at Austin, 2011.

[34] Evans J., Hughes T.: Isogeometric divergence-conforming B-splines for the steady Navier-Stokes equations, Math. Models Methods Appl. Sci. 23(2013), 1421-1478.

[35] Evans J., Hughes T.: Isogeometric divergence-conforming B-splines for the unsteady Navier-Stokes equations, J. Comput. Phys. 241(2013), 141-167.

[36] Falk R.: Nonconforming finite element methods for the equations of linear elasticity, Math. Comput. 57 (1991), 529-550.

[37] Falk R., Morley E.: Equivalence of finite element methods for problems in elasticity, SIAM J. Numer. Anal. 27(1990), 1486-1505.

[38] Falk R., Neilan M.: Stokes complexes and the construction of stable finite elements with pointwise mass conservation, SIAM J. Numer. Anal. 51(2013), 1308-1326.

[39] Faria C., Boness A., Loula A.: A stabilized hybrid finite element method for the linear elasticity problems, in Blucher Mechanical Engineering Proceedings May 2014, vol. 1 , num. 1.

[40] Girault V., Raviart P.: Finite Element Methods for the Navier-Stokes Equations, Springer-Verlag, Berlin, 1986.

[41] Guillén González F., Rodríguez Galván J.R., On the stability of approximations for the Stokes problem using different finite element spaces for each component of the velocity, arXiv:1411.7930v1, 2014.

[42] Guzmán J., Neilan M.: Conforming and divergence-free Stokes elements on general triangular meshes, Math. Comp., in press.

[43] Heys J.J., Lee E., Manteuffel T.A., McCormick S.F.: On mash-conserving least-squares methods, SIAM J. SCI. COMPUT. 28(2006), 1675-1693. 
[44] Hiptmair R., Xu J.: Nodal auxiliary space preconditioning in H(curl) and H(div) spaces, SIAM J. Numer. Anal. 45(2007), 2483-2509.

[45] Hu J.: Locking free quadrilateral elements for elasticity problems, PhD thesis, Academy of Mathematics and System Sciences, Chinese Academy of Sciences, 2004. (in Chinese)

[46] Hu J.: A New Family of Efficient Conforming Mixed Finite Elements on Both Rectangular and Cuboid Meshes for Linear Elasticity in the Symmetric Formulation, SIAM J. Numer. Anal. 53(2015), 1438-1463.

[47] Hu J., Man H., Shi Z.: Constrained nonconforming rotated Q1 element for Stokes and planar elasticity. Math. Numer. Sin. 27(2005), 311-324. (in Chinese)

[48] Hu J., Shi Z.: Constrained nonconforming rotated Q1 element methods of the Reissner?Mindlin plate problem, submitted. (in Chinese)

[49] Huang Y., Zhang S.: A lowest order divergence-free finite element on rectangular grids, Frontiers of Mathematics in China 6(2011), 253-270.

[50] Hughes T., Cottrell J., Bazilevs Y.: Isogeometric analysis: CAD, finite elements, NURBS, exact geometry, and mesh refinement, Comput. Methods Appl. Mech. Engrg. 194(2005), 4135-4195.

[51] Kim S., Yim J., Sheen D.: Stable cheapest nonconforming finite elements for the Stokes equations, J. Compute. Apple. Math., to appear.

[52] Knobloch P., Tobiska L.: On Korn's first inequality for quadrilateral nonconforming finite elements of first order approximation properties, Int. J. Numer. Anal. Model. International 2(2005), 439-458.

[53] Kouhia R., Stenberg R.: A linear nonconforming finite element method for nearly incompressible elasticity and Stokes flow, Comput. Methods Appl. Mech. Engrg. 124(1995), 195-212.

[54] Lamichhane B., Reddy B., Wohlmuth B.: Convergence in the incompressible limit of finite element approximations based on the Hu-Washizu formulation, Numer. Math. 104 (2006), 151-175.

[55] Lin Q., Tobiska L., Zhou A.: Superconvergence and extrapolation of non-conforming low order finite elements applied to the Poisson equation, IMA Journal of Numerical Analysis, 25(2005), 160-181.

[56] Linke A.: Collision in a cross-shaped domain: A steady 2d Navier-Stokes example demonstrating the importance of mass conservation in CFD, Comp. Meth. Appl. Mech. Eng. 198(2009), 3278-3286.

[57] Linke A., Matthies G., Tobiska L.: Non-nested multi-grid solvers for mixed divergence free scott-vogelius discretizations, Computing, 83(2008), 87-107.

[58] Mardal K., Tai X., Winther R.: A robust finite element method for Darcy-Stokes flow, SIAM J. Numer. Anal. 40(2002), 1605-1631.

[59] Ming P., Shi Z.: Two nonconforming quadrilateral elements for the Reissner-Minddlin plate, Math. Models Methods Appl. Sci. 15(2005), 1503-1517.

[60] Nečas J., Hlavàček I.: Mathematical Theory of Elastic and Elastico-Plastic Bodies: An Introduction, Elsevier, Amsterdam, 1981.

[61] Qin J.: On the convergence of some low order mixed finite elements for incompressible fluids, $\mathrm{Ph} . \mathrm{D}$. Thesis, Penn State University, Department of Mathematics, (1994).

[62] Olshanskii M., Reusken A.: Grad-div stabilization for Stokes equations, Math.Comp. 73(2004), 1699-1718.

[63] Scott L., Vogelius M.: Norm estimates for a maximal right inverse of the divergence operator in spaces of piecewise polynomials, RAIRO, Modelisation Math. Anal. Numer. 19(1985), 111-143. 
[64] Scott L., Vogelius L.: Conforming finite element methods for incompressible and nearly incompressible continua, in Lect. Appl. Math., 22 (1985), pp. 221-244.

[65] Shi Z., Wang M.: Finite element methods, Science Press, Beijing, 2013.

[66] Stenberg R.: A technique for analysing finite element methods for viscous incompressible flow, Int. J. Numer. Methods Fluids 11(1990), 935-948.

[67] Vidal Y., Villon P., Huerta A.: Locking in the incompressible limit: pseudo-divergence-free element free Galerkin, Commun. Numer. Methods Engrg. 19(2003), 725-735.

[68] Wang L., Qi H.: A locking-free scheme of nonconforming rectangular finite element for the planar elasticity, J. Comput. Math. 22(2004) 641-650.

[69] Wang M.: The generalized Korn inequality on nonconforming finite element spaces. (in Chinese), Math. Numer. Sinica 16 (1994), no. 1, 108-113; for translation see Chinese J. Numer. Math. Appl. 16 (1994), no. 2, 91-96.

[70] Xie X., Xu J., Xue G.: Uniformly-stable finite element methods for Darcy-Stokes-Brinkman models, Journal of Computational Mathematics 26(2008), 437-455

[71] Xu J., Yang K.: Well-posedness and robust preconditioners for discretized fluid structure interaction systems, Comput. Methods Appl. Mech. Engrg. 292(2015), 69-91.

[72] Zhang S.: Stable finite element pair for Stokes problem and discrete Stokes complex on quadrilateral grids, Numerische Mathematik, to appear.

[73] Zhang S.: A family of $Q_{k+1, k} \times Q_{k, k+1}$ divergence-free finite elements on rectangular grids, SIAM J. Numer. Anal. 47(2009) 2090-2107.

School of Mathematical Science,Peking University, Beijing, 100871, People's Republic of China

E-mail address: 1100010633@pku. edu.cn

LSEC, ICMSEC, NCMis, Academy of Mathematics and System Sciences, Chinese Academy of Sciences, Beijing 100190, People's Republic of China

E-mail address: szhang@lsec.cc.ac.cn 